\title{
Nonforward Compton scattering in AdS/CFT correspondence
}

\author{
Jian-Hua Gad* \\ Department of Modern Physics, University of Science and Technology of China, \\ Hefei, Anhui 230026, People's Republic of China \\ Bo-Wen Xiadt \\ Nuclear Science Division, Lawrence Berkeley National \\ Laboratory, Berkeley, California 94720, USA
}

(Dated: October 22, 2018)

\begin{abstract}
We study the nonforward Compton scattering, in particular, the deeply virtual Compton scattering from AdS/CFT. We first calculate the contributions from the $s$-channel and $u$-channel supergravity diagrams as well as the four-point interaction diagram which correspond to the Compton scatterings on a dilaton target in CFT. Furthermore, we study the Compton scattering on a dilatino target. Assuming that protons can be identified as supergravity modes of the dilatino, we compare the calculated deeply virtual Compton scattering cross section to the low-energy experimental data from the H1 and ZEUS collaborations and find good agreement. We also discuss the $t$-channel graviton exchange contribution and show that it should be dominant in the high-energy limit.
\end{abstract}

PACS numbers: $11.25 . \mathrm{Tq}, 13.60 . \mathrm{Fz}$

*Electronic address: gaojh79@ustc.edu.cn

${ }^{\dagger}$ Electronic address: bxiao@lbl.gov 


\section{INTRODUCTION}

A decade ago, the AdS/CFT correspondence[1 3] was conjectured. Since then, it has provided us with new insights into gauge theories in a strong coupling regime. There has been substantial progress in studying strong coupling gauge theories by applying this technique. In particular, Polchinski and Strassler [4, 5] studied the deep inelastic scattering (DIS) of hadrons by using the correspondence in forward Compton scattering where the usual structure functions $F_{1}$ and $F_{2}$ are calculated for both spinless and spin- $\frac{1}{2}$ hadrons. An infrared cutoff in the fifth dimension at $z=z_{0} \sim 1 / \Lambda_{Q C D}$, which breaks the conformal symmetry in CFT, is introduced to give rise to confinement. In addition, this cutoff scale also provides a mass scale for the hadrons with $M \propto 1 / z_{0}$. This particular model with the hard cutoff in the infrared is then called the hard wall model. There have been a lot of interesting studies [6, 7] and further developments [8 14] along this direction. These studies of DIS and structure functions have provided an interesting picture of hadrons at the strong coupling limit. One finds that there is no large- $x$ parton which carries a finite amount of longitudinal momentum of the target hadron and most of the longitudinal momentum is distributed in the partons sitting in the extremely low- $x$ region. Furthermore, DIS, in terms of dipole (quark-antiquark pair with an open string attached) scattering on a hadron/nucleus target, is discussed in Refs. [15-17]. In particular, the AdS/CFT computation of deep inelastic scattering off the finite temperature plasma has been recently studied in Refs. [1823], which also indicate that most of the constituents in the strongly coupled plasma are located in the very low $x$ region. Based on hard scatterings in AdS/CFT, the saturation picture is then developed in Refs. [24, 25].

Recently, we extended the above DIS calculation in AdS/CFT to the case of polarized DIS in Ref. [26] and obtained the spin-dependent structure functions $g_{1}$ and $g_{2}$ for a spin- $\frac{1}{2}$ hadron. In Ref. [27], the spin decomposition of spin- $\frac{1}{2}$ hadrons at large coupling limit was analyzed. These studies show that the spin of hadrons at large t'Hooft coupling seems solely due to orbital angular momentum. Suggested by Ji[28] in QCD, the deeply virtual Compton scattering (DVCS) can be used as the gateway to the total quark and gluon contributions to the spin of the proton. The DVCS amplitude contains the important information about the total spin of constituents in the hadron which is encoded in the generalized parton distributions. In addition, one can access the orbital angular momentum of quarks and 
gluons by subtracting the spin contributions. Therefore, in order to further investigate the spin and the orbital angular momentum, we need to study the nonforward Compton scattering and extract the so-called generalized parton distributions[28 -37]. As a first step, we study the nonforward Compton scattering, in particular, the DVCS, in the AdS/CFT correspondence. We will leave the extraction of generalized parton distributions for future study.

The objective of this paper is to study the nonforward Compton scattering. We use dilatons (scalar) or dilatinos (fermion) as the targets which are scattered by a virtual gauge boson field (a physical virtual photon, so to speak). We are particularly interested in the DVCS since it is widely studied in QCD and of great importance in spin physics. The final states contain a photon, which is real in DVCS, and the dilaton or dilatino target. This calculation is equivalent to a four-point function calculation that involves two currents $J$ and two targets (dilaton or dilatino) in the AdS/CFT. Four-point functions of the dilaton and axion are studied in Refs. [38-40]. Recently, four-point functions of $\mathcal{R}$-currents are discussed in AdS supergravity [41].

To be more specific, we compute the supergravity graphs corresponding to Compton scattering in CFT. For the case of the dilaton, we have calculated the $s$-channel and $u$ channel graphs where all the Kaluza-Klein excitations of intermediate states are summed over. We have also included a four-point contact term and shown that the sum of these three graphs are gauge invariant, namely, satisfying the current conservation constraint. For the case of the dilatino, we compute the contributions from the $s$-channel and $u$-channel graphs explicitly. Assuming that protons can be identified as supergravity modes of the dilatino, we compare the calculated DVCS cross section to the low-energy H1 and ZEUS data, and find that our calculation is consistent with the data. We also study the real Compton scattering and find that the scattering amplitudes are parametrically the same as the one found in QED.

We notice that there is an additional $t$-channel graviton exchange contribution, which is supposed to be dominant at high energy. We provide a heuristic derivation for the computation of $t$-channel graviton exchange at the end of this paper. Since the graviton exchange only couples to the energy momentum tensor of the target, we find the results for the dilaton and dilatino are quite similar.

The rest of the paper is organized in the following manner: In Sec $\llbracket$ II we consider the 
usual $s$-channel and $u$-channel exchanges as well as the four-point interaction contributions for a dilaton target. In Sec.III, we evaluate the supergravity graphs of the $s$-channel and $u$-channel exchanges for dilatino targets. The $t$-channel exchange of the graviton for both dilaton and dilatino targets is discussed in Sec IV. We summarize and present some more discussion in Sec:V.

\section{COMPTON SCATTERING OF THE DILATON}

In this section, we will formulate the Compton scattering on a scalar target in the CFT.

\section{A. Scaler field in AdS space and its bulk to bulk propagator}

First, let us consider the free scalar field and derive its bulk to bulk propagator in $\mathrm{AdS}_{5}$ space. Scalars in CFT correspond to supergravity modes of dilatons. The metric in $\operatorname{AdS} \times S^{5}$ space can be written as

$$
d s^{2}=\frac{R^{2}}{z^{2}}\left(\eta_{\mu \nu} d y^{\mu} d y^{\nu}+d z^{2}\right)+R^{2} \mathrm{~d} \Omega_{5}^{2},
$$

where $\eta_{\mu \nu}=(-,+,+,+)$ is the mostly plus flat space metric. We assume that the initial/final dilaton wave function can be written as

$$
\Phi(y, z)=e^{i p \cdot y} \phi(z)
$$

where, for simplicity, we have neglected the dependence on the coordinates $S^{5}$. The equation of motion of the dilaton in AdS space can be written as

$$
\frac{1}{\sqrt{-g}} \partial_{m}\left(\sqrt{-g} g^{m n} \partial_{n} \Phi(y, z)\right)-\mu^{2} \Phi(y, z)=0
$$

with the fifth dimension mass $\mu^{2}=\frac{\Delta(\Delta-4)}{R^{2}}$. It reduces to

$$
z^{2} \partial_{z}^{2} \Phi(y, z)-3 z \partial_{z} \Phi(y, z)+z^{2} \square \Phi(y, z)-\Delta(\Delta-4) \Phi(y, z)=0
$$

where $\square=-\partial_{t}^{2}+\nabla^{2}$. The solution to the above equation is given by

$$
\phi(z)=C_{1} z^{2} J_{\Delta-2}(M z)+C_{2} z^{2} Y_{\Delta-2}(M z)
$$

where $M^{2}=-p^{2}$. With the consideration of boundary conditions for normalizable modes, we set $C_{2}=0$ and choose only the $J_{\Delta-2}(M z)$ part. 
In order to calculate the Compton scattering amplitude, we also need the bulk to bulk propagator of dilatons in $A \mathrm{~d} S_{5}$ space, which satisfies

$$
\left[z^{2} \partial_{z}^{2}-3 z \partial_{z}+z^{2} \square-\Delta(\Delta-4)\right] G\left(x, z ; y, z^{\prime}\right)=z^{5} \delta\left(z-z^{\prime}\right) \delta^{(4)}(x-y)
$$

In $\mathrm{AdS}_{5}$ space, from coordinate $(x, z)$ to $\left(y, z^{\prime}\right)$, the propagator can be written as [20, 38],

$$
G\left(x, z ; y, z^{\prime}\right)=-\int \frac{d^{4} k}{(2 \pi)^{4}} e^{-i k \cdot(x-y)} \int_{0}^{\infty} d \omega \frac{\omega}{\omega^{2}+k^{2}-i \epsilon} z^{2} J_{\Delta-2}(\omega z) z^{\prime 2} J_{\Delta-2}\left(\omega z^{\prime}\right),
$$

where $x$ and $y$ are four-dimensional coordinates. One can easily check the above expression satisfies Eq. (5) by noticing that

$$
\left[z^{2} \partial_{z}^{2}-3 z \partial_{z}-\Delta(\Delta-4)\right] z^{2} J_{\Delta-2}(\omega z)=-\omega^{2} z^{2} J_{\Delta-2}(\omega z)
$$

and

$$
\int_{0}^{\infty} d \omega \omega z^{2} J_{\Delta-2}(\omega z) z^{\prime 2} J_{\Delta-2}\left(\omega z^{\prime}\right)=z^{3} \delta\left(z-z^{\prime}\right) .
$$

Note that $k^{2}<0$ corresponds to the timelike case, and $k^{2}>0$ corresponds to spacelike case. When the particle is at rest, we shall have $k^{2}=-M^{2}$. For the case of the $s$-channel Compton scattering, we find that $k=p+q=p^{\prime}+q^{\prime}$. The dilaton propagator in the fifth dimension is then defined as

$$
G\left(z ; z^{\prime}\right)=-\int_{0}^{\infty} d \omega \frac{\omega}{\omega^{2}-M_{X}^{2}-i \epsilon} z^{2} J_{\Delta-2}(\omega z) z^{\prime 2} J_{\Delta-2}\left(\omega z^{\prime}\right)
$$

with $M_{X}^{2}=-(p+q)^{2}$ for the $s$-channel scattering and $M_{X}^{2}=-\left(p-q^{\prime}\right)^{2}$ for the $u$-channel scattering.

\section{B. Kaluza-Klein field in AdS Space}

Following the prescription in Ref. [5], the incident/outgoing current is chosen to be the $\mathcal{R}$ current which couples to the hadron as an isometry of $S^{5}$ with the killing vector $v_{a}$. This is equivalent to saying that the current couples to the target with a conserved charge $\mathcal{Q}$. According to the AdS/CFT correspondence, the current excites a nonnormalizable mode of a Kaluza-Klein gauge field on the Minkowski boundary of the $A d S_{5}$ space

$$
\delta G_{m a}=A_{m}(y, r) v_{a}(\Omega)
$$

This gauge field fluctuation $A_{m}(y, r)$ (a physical virtual photon, so to speak) can be viewed as a vector boson field which couples to the $\mathcal{R}$ current $J^{\mu}$ on the Minkowski boundary, 
and then propagates into the bulk as gravitational waves, and eventually interacts with the supergravity modes of the dilaton or dilatino. The gauge field satisfies Maxwell's equation in the bulk, $D_{m} F^{m n}=0$ which can be explicitly written as

$$
\frac{1}{\sqrt{-g}} \partial_{m}\left[\sqrt{-g} g^{n k} g^{m l}\left(\partial_{k} A_{l}-\partial_{l} A_{k}\right)\right]=0
$$

where $m, n, \ldots$ are indices on $A d S_{5}$. With the boundary condition

$$
A_{\mu}(y, \infty)=\left.A_{\mu}(y)\right|_{4 \mathrm{~d}}=n_{\mu} e^{i q \cdot y}
$$

and the Lorentz-like gauge

$$
\partial_{\mu} A^{\mu}+z \partial_{z}\left(\frac{A_{z}}{z}\right)=0
$$

the Maxwell equation can be written as

$$
\begin{aligned}
-q^{2} A_{\mu}+z \partial_{z}\left(\frac{1}{z} \partial_{z} A_{\mu}\right) & =0 \\
-q^{2} A_{z}+\partial_{z}\left(z \partial_{z}\left(\frac{1}{z} A_{z}\right)\right) & =0 .
\end{aligned}
$$

The solutions to the above equations are given by,

$$
\begin{aligned}
& A_{\mu}=n_{\mu} e^{i q \cdot y} q z K_{1}(q z), \\
& A_{z}=i n \cdot q e^{i q \cdot y} z K_{0}(q z),
\end{aligned}
$$

where $K_{1}$ and $K_{0}$ are both modified Bessel functions and $n_{\mu}$ is the polarization vector. Note that $q^{2}=Q^{2}>0$ for the spacelike current in the mostly plus metric signature.

\section{Compton scattering amplitudes}

Now we are ready to consider the Compton scattering on a dilaton target. The relevant supergravity graphs are shown in Figs. 1 3, corresponding to $s$-channel exchange, $u$-channel exchange and four-point interaction, respectively. The $t$-channel graviton exchange illustrated in Fig. 6 will be considered in Sec.IV.

The standard Compton amplitude is defined as

$$
T_{\mu \nu}=i \int d^{4} y e^{-i q \cdot y}\left\langle P^{\prime}\left|\mathrm{T} J_{\mu}(y) J_{\nu}(0)\right| P\right\rangle .
$$




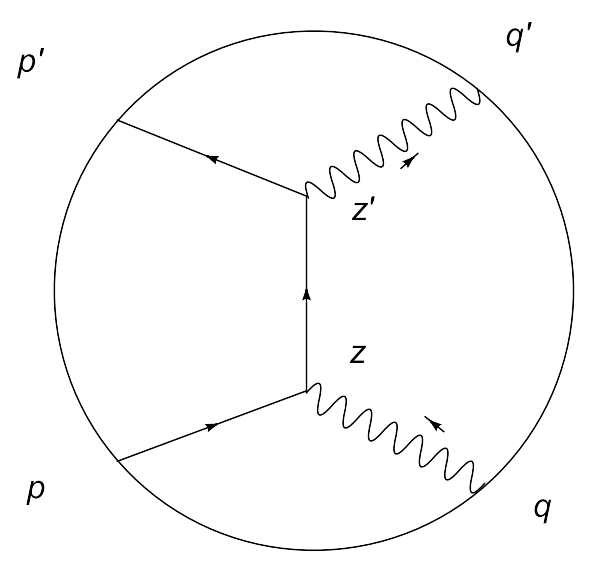

FIG. 1: s-channel Compton scattering

We first start with the calculation of the $s$-channel amplitude as illustrated in Fig. 1, The bulk to bulk propagator from $z$ to $z^{\prime}$ represents high excited states of the dilaton target after absorbing the incoming virtual photon. This is different from the DIS calculation where the propagator is always put on shell. The relevant supergravity interaction of the dilaton and photon is

$$
\begin{aligned}
S_{i n t} & =\int d^{10} x \sqrt{-g} A^{m} v^{a} \partial_{m} \Phi \partial_{a} \Phi \\
& =i \mathcal{Q} \int d^{10} x \sqrt{-g} A^{m}\left(\Phi \partial_{m} \Phi^{*}-\Phi^{*} \partial_{m} \Phi\right)
\end{aligned}
$$

The dilaton is taken to be in a charge eigenstate with the charge $\mathcal{Q}$ under the $U(1)$ symmetry. This yields $v_{a} \partial^{a} \Phi=i \mathcal{Q} \Phi$.

Using the dilaton wave functions along with the bulk to bulk propagator and above interaction vertex, it is straightforward to write down the $s$-channel amplitude

$$
n^{\mu} T_{\mu \nu}^{s} n^{\prime * \nu}=\frac{R^{10}}{R^{8}} \int d^{4} x d^{4} y d z d z^{\prime} \Phi_{i}(x, z) n^{\mu} J_{\mu}(z) G\left(x, z ; y, z^{\prime}\right) n^{\prime * \nu} J_{\nu}^{*}\left(z^{\prime}\right) \Phi_{f}^{*}\left(y, z^{\prime}\right),
$$

where each integration over the $S^{5}$ space gives a factor of $R^{5}$ and the Green's function $G\left(x, z ; y, z^{\prime}\right)$ picks up a factor of $\frac{1}{R^{8}}$ when we take the $S^{5}$ space into account. (One can actually set $R=1$ in the calculation since the final result should not depend on the curvature $R$.) The initial and final wave functions of the dilaton are

$$
\Phi_{i}(x, z)=\frac{c_{i}}{z_{0} R^{4}} z^{2} J_{\Delta-2}(M z) e^{i p \cdot x} \quad \text { and } \quad \Phi_{f}^{*}(y, z)=\frac{c_{f}}{z_{0} R^{4}} z^{2} J_{\Delta-2}(M z) e^{-i p^{\prime} \cdot y},
$$


respectively. The boundary condition is set as $J_{\Delta-2}\left(M z_{0}\right)=0$ in the fifth dimension to mimic confinement. In addition, this cutoff scale also yields a mass scale for the hadrons with $M \propto 1 / z_{0}[42,43]$. It is straightforward to integrate out $x$ and $y$, and then find

$$
n^{\mu} T_{\mu \nu}^{s} n^{\prime * \nu}=(2 \pi)^{4} \delta^{(4)}\left(p+q-p^{\prime}-q^{\prime}\right) R^{2} \int d z d z^{\prime} \Phi_{i}(z) n^{\mu} J_{\mu}(z) G\left(z ; z^{\prime}\right) n^{\prime * \nu} J_{\nu}^{*}\left(z^{\prime}\right) \Phi_{f}^{*}\left(z^{\prime}\right)
$$

The $s$-channel amplitude $T_{\mu \nu}^{s}$ can be cast into

$$
T_{\mu \nu}^{s} \propto\left(2 p_{\mu}+q_{\mu}\right)\left(2 p_{\nu}^{\prime}+q_{\nu}^{\prime}\right) \mathcal{T}_{1}^{s}-\left(2 p_{\mu}+q_{\mu}\right) q_{\nu}^{\prime} \mathcal{T}_{2}^{s}-q_{\mu}\left(2 p_{\nu}^{\prime}+q_{\nu}^{\prime}\right) \mathcal{T}_{3}^{s}+q_{\mu} q_{\nu}^{\prime} \mathcal{T}_{4}^{s}
$$

where

$$
\begin{aligned}
& \mathcal{T}_{1}^{s}=\int d z d z^{\prime} q J_{\Delta-2}(M z) K_{1}(q z) G\left(z, z^{\prime}\right) q^{\prime} J_{\Delta-2}\left(M z^{\prime}\right) K_{1}\left(q^{\prime} z^{\prime}\right) \\
\mathcal{T}_{2}^{s}= & \int d z d z^{\prime} q J_{\Delta-2}(M z) K_{1}(q z) \partial_{z^{\prime}} G\left(z, z^{\prime}\right) J_{\Delta-2}\left(M z^{\prime}\right) K_{0}\left(q^{\prime} z^{\prime}\right) \\
& -\int d z d z^{\prime} q J_{\Delta-2}(M z) K_{1}(q z) G\left(z, z^{\prime}\right) \frac{1}{z^{\prime 2}} \partial_{z^{\prime}}\left[z^{\prime 2} J_{\Delta-2}\left(M z^{\prime}\right)\right] K_{0}\left(q^{\prime} z^{\prime}\right), \\
\mathcal{T}_{3}^{s}= & \int d z d z^{\prime} q J_{\Delta-2}(M z) K_{0}(q z) \partial_{z} G\left(z, z^{\prime}\right) J_{\Delta-2}\left(M z^{\prime}\right) K_{1}\left(q^{\prime} z^{\prime}\right) \\
& -\int d z d z^{\prime} \frac{1}{z^{2}} \partial_{z}\left[z^{2} J_{\Delta-2}(M z)\right] K_{0}(q z) G\left(z, z^{\prime}\right) q^{\prime} J_{\Delta-2}\left(M z^{\prime}\right) K_{1}\left(q^{\prime} z^{\prime}\right),
\end{aligned}
$$

and

$$
\begin{aligned}
\mathcal{T}_{4}^{s}= & \int d z d z^{\prime} J_{\Delta-2}(M z) K_{0}(q z) \partial_{z^{\prime}} \partial_{z} G\left(z, z^{\prime}\right) J_{\Delta-2}\left(M z^{\prime}\right) K_{0}\left(q^{\prime} z^{\prime}\right) \\
& -\int d z d z^{\prime} J_{\Delta-2}(M z) K_{0}(q z) \partial_{z} G\left(z, z^{\prime}\right) \frac{1}{z^{\prime 2}} \partial_{z^{\prime}}\left[z^{\prime 2} J_{\Delta-2}\left(M z^{\prime}\right)\right] K_{0}\left(q^{\prime} z^{\prime}\right) \\
& -\int d z d z^{\prime} K_{0}(q z) \frac{1}{z^{2}} \partial_{z}\left[z^{2} J_{\Delta-2}(M z)\right] \partial_{z^{\prime}} G\left(z, z^{\prime}\right) J_{\Delta-2}\left(M z^{\prime}\right) K_{0}\left(q^{\prime} z^{\prime}\right) \\
& +\int d z d z^{\prime} K_{0}(q z) \frac{1}{z^{2}} \partial_{z}\left[z^{2} J_{\Delta-2}(M z)\right] G\left(z, z^{\prime}\right) \frac{1}{z^{\prime 2}} \partial_{z^{\prime}}\left[z^{\prime 2} J_{\Delta-2}\left(M z^{\prime}\right)\right] K_{0}\left(q^{\prime} z^{\prime}\right) .
\end{aligned}
$$

Using the Eq. (8) and the recurrence relations

$$
\begin{aligned}
& K_{0}(z)=-\frac{d}{d z} K_{1}(z)-\frac{K_{1}(z)}{z}, \\
& J_{\nu}(\alpha z) \frac{d}{d z}\left[z \frac{d}{d z} J_{\nu}(\beta z)\right]-J_{\nu}(\beta z) \frac{d}{d z}\left[z \frac{d}{d z} J_{\nu}(\alpha z)\right]=\left(\alpha^{2}-\beta^{2}\right) z J_{\nu}(\alpha z) J_{\nu}(\beta z),
\end{aligned}
$$

one obtains

$$
\begin{aligned}
& \int d z^{\prime}\left\{\partial_{z^{\prime}} G\left(z, z^{\prime}\right) J_{\Delta-2}\left(M z^{\prime}\right)-\frac{G\left(z, z^{\prime}\right)}{z^{\prime 2}} \partial_{z^{\prime}}\left[z^{\prime 2} J_{\Delta-2}\left(M z^{\prime}\right)\right]\right\} K_{0}\left(q^{\prime} z^{\prime}\right) \\
= & \frac{M^{2}+s}{q^{\prime 2}} \int d z^{\prime} q^{\prime} G\left(z, z^{\prime}\right) J_{\Delta-2}\left(M z^{\prime}\right) K_{1}\left(q^{\prime} z^{\prime}\right)+z^{3} K_{1}\left(q^{\prime} z\right) J_{\Delta-2}(M z) / q^{\prime}
\end{aligned}
$$


where $s=(p+q)^{2}$. Eventually, one can simplify and cast these $\mathcal{T}$ amplitudes into

$$
\begin{aligned}
& \mathcal{T}_{2}^{s}=\left(1-\frac{1}{x^{\prime}}\right) \mathcal{T}_{1}^{s}+\frac{q}{q^{\prime}} \int d z z^{3} J_{\Delta-2}^{2}(M z) K_{1}(q z) K_{1}\left(q^{\prime} z\right), \\
& \mathcal{T}_{3}^{s}=\left(1-\frac{1}{x}\right) \mathcal{T}_{1}^{s}+\frac{q^{\prime}}{q} \int d z z^{3} J_{\Delta-2}^{2}(M z) K_{1}(q z) K_{1}\left(q^{\prime} z\right),
\end{aligned}
$$

and

$$
\begin{aligned}
\mathcal{T}_{4}^{s}= & \left(\frac{1}{x^{\prime}}-1\right)\left(\frac{1}{x}-1\right) \mathcal{T}_{1}^{s}-\int d z z^{3} J_{\Delta-2}^{2}(M z) K_{0}(q z) K_{0}\left(q^{\prime} z\right) \\
& +\frac{M^{2}+s}{q^{\prime} q} \int d z z^{3} J_{\Delta-2}^{2}(M z) K_{1}(q z) K_{1}\left(q^{\prime} z\right)
\end{aligned}
$$

where

$$
x=-\frac{q^{2}}{2 p \cdot q}, \quad x^{\prime}=-\frac{q^{2}}{2 p^{\prime} \cdot q^{\prime}} .
$$

In the end, we obtain the $s$-channel amplitude

$$
\begin{aligned}
T_{\mu \nu}^{s} \propto & \left(2 p_{\mu}+\frac{q_{\mu}}{x}\right)\left(2 p_{\nu}^{\prime}+\frac{q_{\nu}^{\prime}}{x^{\prime}}\right) \mathcal{T}_{1}^{s}-q_{\mu} q_{\nu}^{\prime} \mathcal{C}_{0} \\
& -\left[\left(2 p_{\mu}+q_{\mu}\right) q_{\nu}^{\prime} \frac{q}{q^{\prime}}+q_{\mu}\left(2 p_{\nu}^{\prime}+q_{\nu}^{\prime}\right) \frac{q^{\prime}}{q}-q_{\mu} q_{\nu}^{\prime} \frac{M^{2}+s}{q q^{\prime}}\right] \mathcal{C}_{1}
\end{aligned}
$$

where we have defined that

$$
\begin{aligned}
& \mathcal{C}_{0}=\int d z z^{3} J_{\Delta-2}^{2}(M z) K_{0}(q z) K_{0}\left(q^{\prime} z\right) \\
& \mathcal{C}_{1}=\int d z z^{3} J_{\Delta-2}^{2}(M z) K_{1}(q z) K_{1}\left(q^{\prime} z\right) .
\end{aligned}
$$

Similarly, the amplitude from the $u$-channel diagram, which is illustrated in Fig. 2, is found to be

$$
\begin{aligned}
T_{\mu \nu}^{u} \propto & \left(2 p_{\mu}^{\prime}-\frac{q_{\mu}}{\tilde{x}}\right)\left(2 p_{\nu}-\frac{q_{\nu}^{\prime}}{\tilde{x}^{\prime}}\right) \mathcal{T}_{1}^{u}-q_{\mu} q_{\nu}^{\prime} \mathcal{C}_{0} \\
& +\left[\left(2 p_{\mu}^{\prime}-q_{\mu}\right) q_{\nu}^{\prime} \frac{q}{q^{\prime}}+q_{\mu}\left(2 p_{\nu}-q_{\nu}^{\prime}\right) \frac{q^{\prime}}{q}+q_{\mu} q_{\nu}^{\prime} \frac{M^{2}+u}{q q^{\prime}}\right] \mathcal{C}_{1},
\end{aligned}
$$

where

$$
\tilde{x}=\frac{q^{2}}{2 p^{\prime} \cdot q}, \quad \tilde{x}^{\prime}=\frac{q^{\prime 2}}{2 p \cdot q^{\prime}},
$$

and $u=\left(p^{\prime}-q\right)^{2}$. Note that actually the $u$-channel amplitude and $s$-channel amplitude are related by interchanging $q_{\mu}, n^{\mu}$ and $q_{\nu}^{\prime}, n^{* \nu}$ according to the crossing symmetry. 


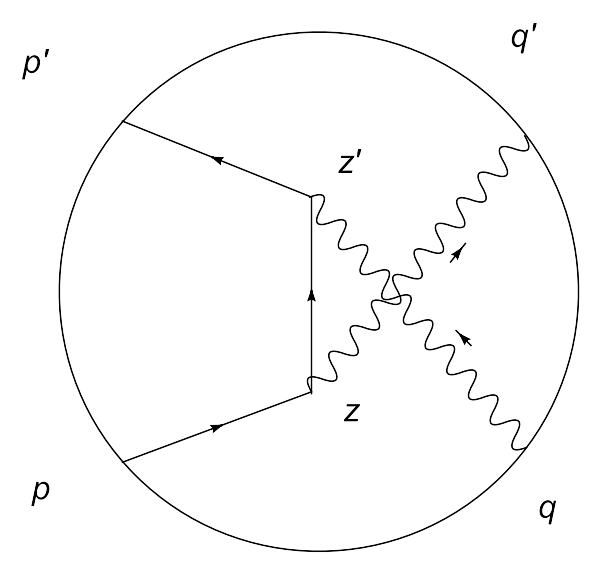

FIG. 2: $u$-channel Compton scattering

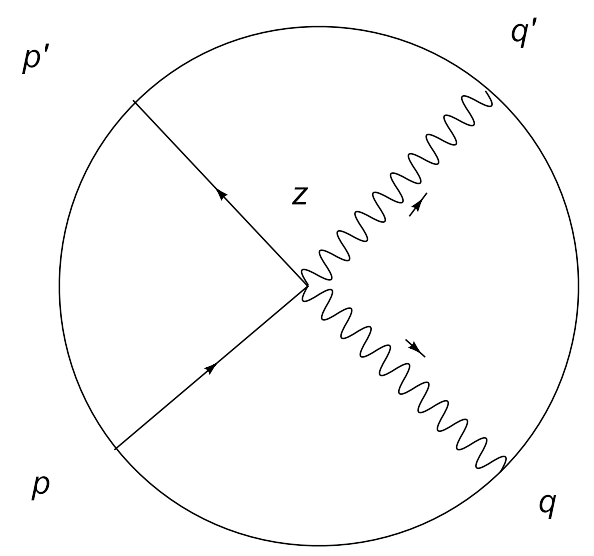

FIG. 3: Four-point interaction of Compton scattering

Last but not least, we need to calculate the four-point interaction contribution, namely, the contact term as depicted in Fig. 3. The four-point interaction amplitude is

$$
n^{\mu} T_{\mu \nu}^{c} n^{\prime * \nu}=2 \mathcal{Q}^{2} R^{5}(2 \pi)^{4} \delta^{(4)}\left(p+q-p^{\prime}-q^{\prime}\right) \int d z \sqrt{-g} \Phi_{i}(z) g^{m n} A_{m} A_{n}^{*} \Phi_{f}^{*}(z) .
$$

Then it is easy to obtain

$$
\begin{aligned}
T_{\mu \nu}^{c} \propto & 2 \eta_{\mu \nu} q^{\prime} q \int d z z^{3} J_{\Delta-2}^{2}(M z) K_{1}(q z) K_{1}\left(q^{\prime} z\right) \\
& +2 q_{\mu} q_{\nu}^{\prime} \int d z z^{3} J_{\Delta-2}^{2}(M z) K_{0}(q z) K_{0}\left(q^{\prime} z\right) .
\end{aligned}
$$

Summing over all the contributions from those supergravity graphs, we arrive at the 
gauge invariant results

$$
\begin{aligned}
T_{\mu \nu}^{\mathrm{tot}}= & T_{\mu \nu}^{s}+T_{\mu \nu}^{u}+T_{\mu \nu}^{c} \\
= & \mathcal{A}\left[\left(2 p_{\mu}+\frac{q_{\mu}}{x}\right)\left(2 p_{\nu}^{\prime}+\frac{q_{\nu}^{\prime}}{x^{\prime}}\right) \mathcal{T}_{1}^{s}+\left(2 p_{\mu}^{\prime}-\frac{q_{\mu}}{\tilde{x}}\right)\left(2 p_{\nu}-\frac{q_{\nu}^{\prime}}{\tilde{x}^{\prime}}\right) \mathcal{T}_{1}^{u}\right] \\
& +2 \mathcal{A}\left(\eta_{\mu \nu} q^{\prime} q-q_{\mu}^{\prime} q_{\nu}^{\prime} \frac{q}{q^{\prime}}-q_{\mu} q_{\nu} \frac{q^{\prime}}{q}+q_{\mu} q_{\nu}^{\prime} \frac{q \cdot q^{\prime}}{q^{\prime} q}\right) \mathcal{C}_{1} .
\end{aligned}
$$

The overall constant is found to be $\mathcal{A}=\frac{\mathcal{Q}^{2} c_{i} c_{f}}{z_{0}^{2}}(2 \pi)^{4} \delta^{(4)}\left(p+q-p^{\prime}-q^{\prime}\right)$. One can easily check the conservation of the current

$$
q^{\mu} T_{\mu \nu}^{\mathrm{tot}}=0 \quad \text { and } \quad T_{\mu \nu}^{\text {tot }} q^{\prime \nu}=0
$$

It is well-known that the DIS amplitude and structure functions can be extracted from the imaginary part of forward Compton scattering amplitude according to the optical theorem. Therefore, one can easily obtain the structure functions $F_{1}$ and $F_{2}$ by taking the forward limit and the imaginary part of $T_{\mu \nu}^{\mathrm{tot}}$. The results agree with those found in Ref. [5] in the large- $x$ region. The same conclusion can be also drawn for a dilatino target.

\section{Real Compton scattering}

In the case of real Compton scattering, it follows that $q^{2}=q^{2}=0$ since the incoming and outgoing photons are taken to be real photons. Since a real photon only has 2 degrees of freedom, one can choose a polarization vector $\varepsilon^{\mu}(q, \lambda)$ which is orthogonal to $p_{\mu}$ and $q_{\mu}$ in a special gauge. Similarly we can also choose to have $\varepsilon^{\prime \nu}\left(q^{\prime}, \lambda^{\prime}\right) q_{\nu}^{\prime}=0$ and $\varepsilon^{\prime \nu}\left(q^{\prime}, \lambda^{\prime}\right) p_{\nu}=0$ for the outgoing photon in the target rest frame. Therefore, the amplitude of real Compton scattering can be reduced to

$$
\mathcal{M}=2 \mathcal{Q}^{2} c_{i} c_{f} \varepsilon(q, \lambda) \cdot \varepsilon^{\prime}\left(q^{\prime}, \lambda^{\prime}\right) B
$$

with $B=\frac{1}{z_{0}^{2}} \int_{0}^{z_{0}} d z z J_{\Delta-2}^{2}(M z)=\frac{1}{2}\left[J_{\Delta-2}^{2}\left(M z_{0}\right)-J_{\Delta-1}\left(M z_{0}\right) J_{\Delta-3}\left(M z_{0}\right)\right]>0$ when $z_{0}>0$. This eventually leads to the unpolarized Compton cross section after averaging over $\lambda$ and summing over $\lambda^{\prime}$ which gives $\frac{1}{2} \sum_{\lambda, \lambda^{\prime}}\left|\varepsilon(q, \lambda) \cdot \varepsilon^{\prime}\left(q^{\prime}, \lambda^{\prime}\right)\right|^{2}=\frac{1}{2}\left(1+\cos ^{2} \theta\right)$. Thus eventually one gets

$$
\frac{d \sigma^{\mathrm{Un}}}{d \Omega_{q^{\prime}}} \propto \mathcal{Q}^{4} \frac{q_{0}^{\prime 2}}{q_{0}^{2}}\left(1+\cos ^{2} \theta\right),
$$


where $\theta$ is the angle between the incoming photon and outgoing photon. This result ${ }^{1}$ is identical to the cross section found in scalar electrodynamics except for the overall constant. ${ }^{2}$

\section{E. Deeply virtual Compton scattering}

In the case of deeply virtual Compton scattering, only the final state photon is real. Therefore, we set $q^{\prime 2}=0$ but assume $q^{2}$ is large as compared to $M^{2}$. Hereafter in this subsection, we work in a limit that $M \rightarrow 0$ and $z_{0} \rightarrow \infty$ but with $M z_{0}$ fixed. In this case, the outgoing real photon has 2 degrees of freedom, while the incoming virtual photon has 3. It is usually convenient to choose to stay in the target rest frame (the lab frame). In this frame, we can choose to have $\varepsilon^{\prime \nu}\left(q^{\prime}, \lambda^{\prime}\right) q_{\nu}^{\prime}=0$ and $\varepsilon^{\prime \nu}\left(q^{\prime}, \lambda^{\prime}\right) p_{\nu}=0$ as well as $\varepsilon^{\mu}(q, \lambda) q_{\mu}=0$. This yields

$$
\mathcal{M}=2 \mathcal{Q}^{2} \frac{c_{i} c_{f}}{z_{0}^{2}} \varepsilon(q, \lambda) \cdot \varepsilon^{\prime}\left(q^{\prime}, \lambda^{\prime}\right) \mathcal{C}_{1 \mathrm{DVCS}}+4 \mathcal{Q}^{2} \frac{c_{i} c_{f}}{z_{0}^{2}} \varepsilon(q, \lambda) \cdot p \varepsilon^{\prime}\left(q^{\prime}, \lambda^{\prime}\right) \cdot p^{\prime} \mathcal{T}_{1 \mathrm{DVCS}}^{s}
$$

where

$$
\begin{aligned}
\mathcal{C}_{1 \mathrm{DVCS}} & =\int_{0}^{z_{0}} d z z^{2} J_{\Delta-2}^{2}(M z) q K_{1}(q z) \\
& \simeq \frac{2(\Delta-1)}{M^{2}}\left(\frac{M^{2}}{q^{2}}\right)^{\Delta-1}{ }_{2} F_{1}\left(\Delta-\frac{3}{2}, \Delta, 2 \Delta-3,-\frac{4 M^{2}}{q^{2}}\right)
\end{aligned}
$$

and

$$
\begin{aligned}
\mathcal{T}_{1 \mathrm{DVCS}}^{s}= & -\int_{0}^{\infty} d \omega \frac{\omega}{\omega^{2}+(p+q)^{2}-i \epsilon} \int_{0}^{z_{0}} d z q J_{\Delta-2}(M z) K_{1}(q z) z^{2} J_{\Delta-2}(\omega z) \\
& \times \int_{0}^{z_{0}} d z^{\prime} z^{\prime} J_{\Delta-2}\left(\omega z^{\prime}\right) J_{\Delta-2}\left(M z^{\prime}\right) \\
\simeq & \frac{-1}{M^{2}+(p+q)^{2}-i \epsilon} \frac{2(\Delta-1)}{M^{2}}\left(\frac{M^{2}}{q^{2}}\right)^{\Delta-1}{ }_{2} F_{1}\left(\Delta-\frac{3}{2}, \Delta, 2 \Delta-3,-\frac{4 M^{2}}{q^{2}}\right),
\end{aligned}
$$

where we approximately write $\int_{0}^{z_{0}} d z^{\prime} z^{\prime} J_{\Delta-2}\left(\omega z^{\prime}\right) J_{\Delta-2}\left(M z^{\prime}\right) \simeq \frac{1}{M} \delta(\omega-M)$, which is an exact result if $z_{0} \rightarrow \infty$. One can approximately write $\mathcal{T}_{1 \mathrm{DVCS}}^{s} \simeq \frac{-1}{M^{2}+(p+q)^{2}-i \epsilon} \mathcal{C}_{1 \mathrm{DVCS}}$.

\footnotetext{
${ }^{1}$ The real Compton amplitude is sensitive to the IR region of the target wave function. For a different geometry other than AdS, we expect that Eq. (41) will still hold while the constant $B$ may change since it depends on the details of the IR cutoff.

2 Here we do not take the $t$-channel graviton exchange contribution into account since it belongs to another class of contributions. The discussion on this issue is given in the end of Sec. III and the calculation of $t$-channel graviton exchange amplitude is provided in Sec. IV.
} 
To obtain the cross section, let us work out the kinematics first. We analyze the deeply virtual Compton scattering in the lab frame, in which the initial scalar is at rest. Thus,

$$
\begin{aligned}
& \text { Before the scattering } p_{\mu}=(M, 0,0,0), q_{\mu}=\left(q_{0}, 0,0, q_{3}\right) ; \\
& \text { After the scattering } p_{\mu}^{\prime}=\left(p_{0}^{\prime}, p_{1}^{\prime}, 0, p_{3}^{\prime}\right), q_{\mu}^{\prime}=\left(\omega^{\prime}, \omega^{\prime} \sin \theta, 0, \omega^{\prime} \cos \theta\right) .
\end{aligned}
$$

According to the conservation of energy momentum, it is straightforward to see that

$$
\begin{aligned}
& p_{0}^{\prime}=M+q_{0}-\omega^{\prime}, \\
& p_{1}^{\prime}=-\omega^{\prime} \sin \theta, \\
& p_{3}^{\prime}=q_{3}-\omega^{\prime} \cos \theta .
\end{aligned}
$$

In addition, we know that

$$
q^{2}=q_{3}^{2}-q_{0}^{2} \text { and } p^{\prime 2}=-M^{2}
$$

which lead us to

$$
\omega^{\prime}=\frac{2 M q_{0}-q^{2}}{2 q_{0}+2 M-2 q_{3} \cos \theta} .
$$

We write the polarization vectors explicitly in the following way:

$$
\begin{aligned}
\varepsilon^{\mu}(q, 0) & =\frac{1}{q}\left(q_{3}, 0,0, q_{0}\right), \\
\varepsilon^{\mu}(q,+1) & =(0,1,0,0), \\
\varepsilon^{\mu}(q,-1) & =(0,0,1,0), \\
\varepsilon^{\prime \mu}(q,+1) & =(0, \cos \theta, 0,-\sin \theta), \\
\varepsilon^{\prime \mu}(q,-1) & =(0,0,1,0),
\end{aligned}
$$

where all the transverse polarization vectors are normalized to 1 . Therefore, one can easily average over $\lambda$ and sum over $\lambda^{\prime}$, which yields

$$
\begin{aligned}
\frac{1}{2} \sum_{\lambda, \lambda^{\prime}}|\mathcal{M}|^{2} & =2 \mathcal{Q}^{4} \frac{c_{i}^{2} c_{f}^{2}}{z_{0}^{4}} \mathcal{C}_{1 \mathrm{DVCS}}^{2} \sum_{\lambda, \lambda^{\prime}}\left|\varepsilon(q, \lambda) \cdot \varepsilon^{\prime}\left(q^{\prime}, \lambda^{\prime}\right)\right|^{2} \\
& +8 \mathcal{Q}^{4} \frac{c_{i}^{2} c_{f}^{2}}{z_{0}^{4}} \mathcal{T}_{1 \mathrm{DVCS}}^{s 2} \sum_{\lambda, \lambda^{\prime}}\left|\varepsilon(q, \lambda) \cdot p \varepsilon^{\prime}\left(q^{\prime}, \lambda^{\prime}\right) \cdot p^{\prime}\right|^{2} \\
& +8 \mathcal{Q}^{4} \frac{c_{i}^{2} c_{f}^{2}}{z_{0}^{4}} \mathcal{C}_{1 \mathrm{DVCS}} \mathcal{T}_{1 \mathrm{DVCS}}^{s} \sum_{\lambda, \lambda^{\prime}} \varepsilon(q, \lambda) \cdot p \varepsilon^{\prime}\left(q^{\prime}, \lambda^{\prime}\right) \cdot p^{\prime} \varepsilon(q, \lambda) \cdot \varepsilon^{\prime}\left(q^{\prime}, \lambda^{\prime}\right) .
\end{aligned}
$$


With the above polarization vectors, we find

$$
\begin{aligned}
& \sum_{\lambda, \lambda^{\prime}}\left|\varepsilon(q, \lambda) \cdot \varepsilon^{\prime}\left(q^{\prime}, \lambda^{\prime}\right)\right|^{2}=1+\cos ^{2} \theta+\frac{q_{0}^{2}}{q^{2}} \sin ^{2} \theta, \\
& \sum_{\lambda, \lambda^{\prime}}\left|\varepsilon(q, \lambda) \cdot p \varepsilon^{\prime}\left(q^{\prime}, \lambda^{\prime}\right) \cdot p^{\prime}\right|^{2}=M^{2} q_{3}^{2} \frac{q_{3}^{2}}{q^{2}} \sin ^{2} \theta, \\
& \sum_{\lambda, \lambda^{\prime}}[\varepsilon(q, \lambda) \cdot p]\left[\varepsilon^{\prime}\left(q^{\prime}, \lambda^{\prime}\right) \cdot p^{\prime}\right]\left[\varepsilon(q, \lambda) \cdot \varepsilon^{\prime}\left(q^{\prime}, \lambda^{\prime}\right)\right]=-M q_{0} \frac{q_{3}^{2}}{q^{2}} \sin ^{2} \theta .
\end{aligned}
$$

Therefore, the averaged amplitude can be written as

$$
\begin{aligned}
\frac{1}{2} \sum_{\lambda, \lambda^{\prime}}|\mathcal{M}|^{2} \simeq & 2 \mathcal{Q}^{4} \frac{c_{i}^{2} c_{f}^{2}}{z_{0}^{4}} \mathcal{C}_{1 \mathrm{DVCS}}^{2}\left[1+\cos ^{2} \theta+\frac{q_{0}^{2}}{q^{2}} \sin ^{2} \theta\right] \\
& +8 \mathcal{Q}^{4} \frac{c_{i}^{2} c_{f}^{2}}{z_{0}^{4}} \mathcal{C}_{1 \mathrm{DVCS}}^{2}\left[\frac{4 M^{2} q_{3}^{2}}{\left(M^{2}+(p+q)^{2}\right)^{2}}+\frac{4 M q_{0}}{M^{2}+(p+q)^{2}}\right] \frac{q_{3}^{2}}{q^{2}} \sin ^{2} \theta
\end{aligned}
$$

The phase space integral in this frame is

$$
\int d \Pi_{2}=\frac{1}{4 \pi} \frac{\omega^{\prime 2}}{2 M q_{0}-q^{2}} \int d \cos \theta .
$$

Plugging everything into the cross-section formula, one finds

$$
\begin{aligned}
\frac{d \sigma^{\mathrm{Un}}}{d \cos \theta} & =\frac{1}{2 q_{0}} \frac{1}{2 M} \frac{1}{4 \pi} \frac{\omega^{\prime 2}}{2 M q_{0}-q^{2}} \frac{1}{2} \sum_{\lambda, \lambda^{\prime}}|\mathcal{M}|^{2} \\
& =\frac{\omega^{\prime 2}}{16 M^{2} q_{0}^{2}(1-x)} \mathcal{Q}^{4} \frac{c_{i}^{2} c_{f}^{2}}{z_{0}^{4}} \mathcal{C}_{1 \mathrm{DVCS}}^{2}\left[1+\cos ^{2} \theta+\chi \sin ^{2} \theta\right]
\end{aligned}
$$

with

$$
\begin{aligned}
\chi & =\frac{q_{0}^{2}}{q^{2}}+\frac{q_{3}^{2}}{q^{2}}\left[\frac{1}{(1-x)^{2}} \frac{q_{3}^{2}}{q_{0}^{2}}-\frac{2}{1-x}\right] \\
& =\frac{q^{2}}{4 M^{2}(1-x)^{2}}\left(1+\frac{4 M^{2}}{q^{2}} x\right)^{2} .
\end{aligned}
$$

When $q^{2} \gg 4 M^{2}$, one finds $\chi \gg 1$.

\section{COMPTON SCATTERING OF DILATINO}

In this section, we formulate the Compton scattering on a fermion target by evaluating the $s$-channel and $u$-channel supergravity diagrams. 


\section{A. Fermion field in AdS space and bulk to bulk propagator}

Spin- $\frac{1}{2}$ hadrons correspond to supergravity modes of the dilatino which satisfy the Dirac equation in $\mathrm{AdS}_{5}$ space. In the conformal region the dilatino field can be written as

$$
\lambda=\Psi(y, z) \otimes \eta(\Omega)
$$

where $\Psi(y, z)$ is an $S O(4,1)$ spinor on $\mathrm{AdS}_{5}$ and $\eta(\Omega)$ is an $S O(5)$ spinor on $S^{5}$. The wave function $\psi$ satisfies a five-dimensional Dirac equation in AdS space. Let us first derive the five-dimensional Dirac equation in the following.

Since the Dirac gamma matrices are defined in the flat spacetime, we need to use vielbeins to convert them to curved spacetime and make the product $\not D$ Lorentz invariant. A convenient choice of such vielbeins is given by

$$
e_{m}^{a}=\frac{R}{z} \delta_{m}^{a}, \quad e^{m a}=\frac{z}{R} \eta^{m a} \text { and } e_{a}^{m}=\frac{z}{R} \delta_{a}^{m},
$$

where $m=0,1,2,3,5$. The Levi-Civita connection, which is also known as the Christoffel connection, is given by

$$
\Gamma_{m n}^{p}=\frac{1}{2} g^{p q}\left(\partial_{n} g_{m q}+\partial_{m} g_{n q}-\partial_{q} g_{m n}\right)
$$

Here we use $a, b, c$ to denote indices in flat space, and $m, n, p, q$ to denote indices in curved space $\left(\mathrm{AdS}_{5}\right.$ space). In addition, the Greek indices $\mu, \nu$ are defined in Minkowski space. From the metric, one knows

$$
g_{m n}=\frac{R^{2}}{z^{2}} \eta_{m n}
$$

It is then straightforward to work out the Levi-Civita connection in $\mathrm{AdS}_{5}$ space

$$
\Gamma_{\mu \nu}^{5}=\frac{1}{z} \eta_{\mu \nu}, \quad \Gamma_{55}^{5}=-\frac{1}{z} \text { and } \Gamma_{\nu 5}^{\mu}=-\frac{1}{z} \delta_{\nu}^{\mu}
$$

From the above vielbeins and Levi-Civita connections, we can compute the spin connection which is defined as

$$
\omega_{m}^{a b}=e_{n}^{a} \partial_{m} e^{n b}+e_{n}^{a} e^{p b} \Gamma_{p m}^{n}
$$

The only nonvanishing spin connections are

$$
\omega_{\mu}^{5 \nu}=-\omega_{\mu}^{\nu 5}=\frac{1}{z} \delta_{\mu}^{\nu}
$$

Using the above results, the operator $\not D$ can be cast into

$$
\not D=g^{m n} e_{n}^{a} \gamma_{a}\left(\partial_{m}+\frac{1}{2} \omega_{m}^{b c} \Sigma_{b c}\right)=\frac{z}{R}\left(\gamma^{5} \partial_{z}+\gamma^{\mu} \partial_{\mu}-\frac{2}{z} \gamma^{5}\right),
$$


with $\Sigma_{\mu 5}=\frac{1}{4}\left[\gamma_{\mu}, \gamma_{5}\right] .{ }^{3}$ The free dilatino field in $\mathrm{AdS}_{5}$ space satisfies the Dirac equation [44, 45]

$$
(\not D-m) \Psi=\frac{z}{R}\left(\gamma^{5} \partial_{z}+\gamma^{\mu} \partial_{\mu}-\frac{2}{z} \gamma^{5}-\frac{m R}{z}\right) \Psi=0 .
$$

Its normalizable solution is given by

$$
\Psi(z, y)=C e^{i p \cdot y} z^{\frac{5}{2}}\left[J_{m R-1 / 2}(M z) P_{+}+J_{m R+1 / 2}(M z) P_{-}\right] u_{\sigma}
$$

with

$$
\not p u_{\sigma}=-i M u_{\sigma}(\sigma=1,2), \quad M^{2}=-p^{2}, \quad P_{ \pm}=\frac{1}{2}\left(1 \pm \gamma^{5}\right) .
$$

Therefore, we choose the initial and final wave functions of the dilatino to be

$$
\begin{aligned}
\Psi_{i}(y, z) & =\frac{c_{i}^{\prime}}{z_{0} R^{9 / 2}} e^{i p \cdot y} z^{5 / 2}\left[J_{m R-1 / 2}(M z) P_{+}+J_{m R+1 / 2}(M z) P_{-}\right] u_{i \sigma} \\
\bar{\Psi}_{f}^{*}\left(y^{\prime}, z^{\prime}\right) & =\frac{c_{f}^{\prime}}{z_{0} R^{9 / 2}} e^{-i p^{\prime} \cdot y^{\prime}} \bar{u}_{f \sigma^{\prime}} z^{\prime 5 / 2}\left[J_{m R-1 / 2}\left(M z^{\prime}\right) P_{-}+J_{m R+1 / 2}\left(M z^{\prime}\right) P_{+}\right],
\end{aligned}
$$

with a chosen boundary condition $J_{m R-1 / 2}\left(M z_{0}\right)=0$.

Furthermore, we can now consider the bulk to bulk propagator of dilatino. It satisfies the following differential equation

$$
\frac{z}{R}\left(\gamma^{5} \partial_{z}+\gamma^{\mu} \partial_{\mu}-\frac{2}{z} \gamma^{5}-\frac{m R}{z}\right) G\left(z, y ; z^{\prime}, y^{\prime}\right)=\frac{z^{5}}{R} \delta\left(z-z^{\prime}\right) \delta^{4}\left(y-y^{\prime}\right) .
$$

Using the identity

$$
\begin{aligned}
& (\not D-m)\left\{e^{i k \cdot y} z^{\frac{5}{2}}\left[J_{m R-1 / 2}(\omega z) P_{+}+J_{m R+1 / 2}(\omega z) P_{-}\right]\right\} \\
= & \frac{z}{R}\left\{e^{i p \cdot y} z^{\frac{5}{2}}\left[J_{m R-1 / 2}(\omega z) P_{-}+J_{m R+1 / 2}(\omega z) P_{+}\right]\right\}(i \not k-\omega),
\end{aligned}
$$

it is to straightforward to verify that the solution of Eq. (81) is given by

$$
\begin{aligned}
G\left(y, z ; y^{\prime}, z^{\prime}\right)= & -\int \frac{d^{4} k}{(2 \pi)^{4}} e^{-i k \cdot\left(y-y^{\prime}\right)} \int_{0}^{\infty} d \omega \omega z^{5 / 2}\left[J_{m R-1 / 2}(\omega z) P_{+}+J_{m R+1 / 2}(\omega z) P_{-}\right] \\
& \times \frac{-i \not k+\omega}{\omega^{2}+k^{2}-i \epsilon} z^{\prime 5 / 2}\left[J_{m R-1 / 2}\left(\omega z^{\prime}\right) P_{-}+J_{m R+1 / 2}\left(\omega z^{\prime}\right) P_{+}\right] .
\end{aligned}
$$

Following the usual convention, we start with the final state fermion and end with the initial state fermion when we write the amplitude. Thus the following Green's function is

\footnotetext{
${ }^{3}$ We define the $\gamma$-matrices according to the Dirac algebra in the mostly plus metric signature -+++ . The $\gamma_{\mu}$ that we used differ from the conventional gamma matrices by a factor of $i$.
} 
used hereafter:

$$
\begin{aligned}
G\left(z^{\prime}, y^{\prime} ; z, y\right)= & -\int \frac{d^{4} k}{(2 \pi)^{4}} e^{i k \cdot\left(y^{\prime}-y\right)} \int_{0}^{\infty} d \omega \omega z^{5 / 2}\left[J_{m R-1 / 2}\left(\omega z^{\prime}\right) P_{+}+J_{m R+1 / 2}\left(\omega z^{\prime}\right) P_{-}\right] \\
& \times \frac{i \not k+\omega}{\omega^{2}+k^{2}-i \epsilon} z^{5 / 2}\left[J_{m R-1 / 2}(\omega z) P_{-}+J_{m R+1 / 2}(\omega z) P_{+}\right] .
\end{aligned}
$$

\section{B. Compton scattering amplitudes}

The interaction vertex between the Kaluza-Klein gauge field and dilatino is given by

$$
\begin{aligned}
S_{i n t} & =\int d^{10} x \sqrt{-g} A_{m} v^{w} \bar{\lambda}_{X} \gamma^{m} \partial_{w} \lambda_{i} \\
& =i \mathcal{Q} R^{5} \int d^{5} x \sqrt{-g} A_{m} \bar{\Psi}_{X}^{*} e^{m}{ }_{a} \gamma^{a} \Psi_{i}
\end{aligned}
$$

where $\lambda$ stands for the full 10-dimensional wave function of the dilatino state. In arriving at the above expression, we have used the fact that $v^{w} \partial_{w} \lambda_{i}=i \mathcal{Q} \lambda_{i}$ and the integration over $S^{5}$ gives $R^{5}$.

Now we are ready to calculate the Compton scattering amplitude of the dilatino from gauge/string duality. In this section, we consider the following two diagrams, namely, the $s$-channel and $u$-channel graphs. The $s$-channel is illustrated in Fig. 1. Here we use the bulk to bulk propagator from $z^{\prime}$ to $z$, which is different from the deeply inelastic scattering where the propagator is always taken to be on shell. Using the dilatino bulk to bulk propagator and above interaction vertex, we can write $s$-channel amplitude $T_{\mu \nu}^{s}$ as

$$
T_{\mu \nu}^{s}=T_{\mu \nu 1}^{s}+T_{\mu \nu 2}^{s}+T_{\mu \nu 3}^{s}+T_{\mu \nu 4}^{s}
$$

where

$$
\begin{aligned}
T_{1 \mu \nu}^{s}= & \mathcal{A}^{\prime} \int d z \int d z^{\prime} z^{-1 / 2} z^{\prime-1 / 2} q^{\prime} K_{1}\left(q^{\prime} z^{\prime}\right) q K_{1}(q z) \\
& \times \bar{u}_{\sigma^{\prime}}\left(p^{\prime}\right)\left[J_{m R-1 / 2}\left(M z^{\prime}\right) P_{-}+J_{m R+1 / 2}\left(M z^{\prime}\right) P_{+}\right] \gamma_{\mu} \\
& \times G\left(z^{\prime}, z ; p+q\right) \gamma_{\nu}\left[J_{m R-1 / 2}(M z) P_{+}+J_{m R+1 / 2}(M z) P_{-}\right] u_{\sigma}(p), \\
T_{2 \mu \nu}^{s}= & i \mathcal{A}^{\prime} \int d z \int d z^{\prime} z^{-1 / 2} z^{\prime-1 / 2} q^{\prime} K_{1}\left(q^{\prime} z^{\prime}\right) q_{\nu} K_{0}(q z) \\
& \times \bar{u}_{\sigma^{\prime}}\left(p^{\prime}\right)\left[J_{m R-1 / 2}\left(M z^{\prime}\right) P_{-}+J_{m R+1 / 2}\left(M z^{\prime}\right) P_{+}\right] \gamma_{\mu} \\
& \times G\left(z^{\prime}, z ; p+q\right) \gamma^{5}\left[J_{m R-1 / 2}(M z) P_{+}+J_{m R+1 / 2}(M z) P_{-}\right] u_{\sigma}(p),
\end{aligned}
$$




$$
\begin{aligned}
T_{3 \mu \nu}^{s}= & -i \mathcal{A}^{\prime} \int d z \int d z^{\prime} z^{-1 / 2} z^{\prime-1 / 2} q_{\mu}^{\prime} K_{0}\left(q^{\prime} z^{\prime}\right) q K_{1}(q z) \\
& \times \bar{u}_{\sigma^{\prime}}\left(p^{\prime}\right)\left[J_{m R-1 / 2}\left(M z^{\prime}\right) P_{-}+J_{m R+1 / 2}\left(M z^{\prime}\right) P_{+}\right] \gamma^{5} \\
& \times G\left(z^{\prime}, z ; p+q\right) \gamma_{\nu}\left[J_{m R-1 / 2}(M z) P_{+}+J_{m R+1 / 2}(M z) P_{-}\right] u_{\sigma}(p), \\
T_{4 \mu \nu}^{s}= & \mathcal{A}^{\prime} \int d z \int d z^{\prime} z^{-1 / 2} z^{\prime-1 / 2} q_{\mu}^{\prime} K_{0}\left(q^{\prime} z^{\prime}\right) q_{\nu} K_{0}(q z) \\
& \times \bar{u}_{\sigma^{\prime}}\left(p^{\prime}\right)\left[J_{m R-1 / 2}\left(M z^{\prime}\right) P_{-}+J_{m R+1 / 2}\left(M z^{\prime}\right) P_{+}\right] \gamma^{5} \\
& \times G\left(z^{\prime}, z ; p+q\right) \gamma^{5}\left[J_{m R-1 / 2}(M z) P_{+}+J_{m R+1 / 2}(M z) P_{-}\right] u_{\sigma}(p),
\end{aligned}
$$

with $\mathcal{A}^{\prime}=\frac{\mathcal{Q}^{2} c_{c}^{\prime} c_{f}^{\prime}}{z_{0}^{2}}(2 \pi)^{4} \delta^{(4)}\left(p+q-p^{\prime}-q^{\prime}\right)^{4}$ and

$$
\begin{aligned}
G\left(z^{\prime}, z ; k\right)= & -\int_{0}^{\infty} d \omega \omega z^{15 / 2}\left[J_{m R-1 / 2}\left(\omega z^{\prime}\right) P_{+}+J_{m R+1 / 2}\left(\omega z^{\prime}\right) P_{-}\right] \\
& \times \frac{i \not k+\omega}{\omega^{2}+k^{2}-i \epsilon} z^{5 / 2}\left[J_{m R-1 / 2}(\omega z) P_{-}+J_{m R+1 / 2}(\omega z) P_{+}\right] .
\end{aligned}
$$

Using Eq. (25) and $\not p u_{\sigma}=-i M u_{\sigma}$, along with the recurrence relations

$$
\begin{aligned}
\frac{d}{d x} J_{n}(x) & =J_{n-1}(x)-\frac{n}{x} J_{n}(x), \\
\frac{d}{d x} J_{n}(x) & =\frac{n}{x} J_{n}(x)-J_{n+1}(x),
\end{aligned}
$$

we can have

$$
\begin{aligned}
& \int d z z^{2} K_{0}(q z)(i \not k+\omega)\left[J_{m R-1 / 2}(\omega z) P_{-}+J_{m R+1 / 2}(\omega z) P_{+}\right] \\
& \times \gamma^{5}\left[J_{m R-1 / 2}(M z) P_{+}+J_{m R+1 / 2}(M z) P_{-}\right] u_{\sigma}(p) \\
= & \int d z z^{2} q K_{1}(q z)(i \not k+\omega)\left[J_{m R-1 / 2}(\omega z) P_{-}+J_{m R+1 / 2}(\omega z) P_{+}\right] \frac{i \phi}{q^{2}} \\
& \times\left[J_{m R-1 / 2}(M z) P_{+}+J_{m R+1 / 2}(M z) P_{-}\right] u_{\sigma}(p) \\
& +\int d z z^{2} q K_{1}(q z) \frac{\omega^{2}+k^{2}}{q^{2}}\left[J_{m R-1 / 2}(\omega z) P_{+}+J_{m R+1 / 2}(\omega z) P_{-}\right] \\
& \times\left[J_{m R-1 / 2}(M z) P_{+}+J_{m R+1 / 2}(M z) P_{-}\right] u_{\sigma}(p) .
\end{aligned}
$$

Similarly, we can also find

$$
\int d z z^{2} K_{0}\left(q^{\prime} z\right) \bar{u}_{\sigma^{\prime}}\left(p^{\prime}\right)\left[J_{m R-1 / 2}(M z) P_{-}+J_{m R+1 / 2}(M z) P_{+}\right]
$$

\footnotetext{
${ }^{4}$ Here we assign each dilatino propagator a factor of $\frac{1}{R^{9}}$, and find that the Compton amplitude is independent of $R$.
} 


$$
\begin{aligned}
& \times \gamma^{5}\left[J_{m R-1 / 2}(\omega z) P_{+}+J_{m R+1 / 2}(\omega z) P_{-}\right](i \not k+\omega) \\
= & -\int d z z^{2} q^{\prime} K_{1}\left(q^{\prime} z\right) \bar{u}_{\sigma^{\prime}}\left(p^{\prime}\right)\left[J_{m R-1 / 2}(M z) P_{-}+J_{m R+1 / 2}(M z) P_{+}\right] \\
& \times \frac{i q^{\prime}}{q^{\prime 2}}\left[J_{m R-1 / 2}(\omega z) P_{+}+J_{m R+1 / 2}(\omega z) P_{-}\right](i \not k+\omega) \\
& -\int d z z^{2} q^{\prime} K_{1}\left(q^{\prime} z\right) \bar{u}_{\sigma^{\prime}}\left(p^{\prime}\right)\left[J_{m R-1 / 2}(M z) P_{-}+J_{m R+1 / 2}(M z) P_{+}\right] \\
& \times\left[J_{m R-1 / 2}(\omega z) P_{-}+J_{m R+1 / 2}(\omega z) P_{+}\right] \frac{\omega^{2}+k^{2}}{q^{\prime 2}} .
\end{aligned}
$$

This can simplify the Compton scattering amplitude a lot, and lead to

$$
\begin{aligned}
T_{2 \mu \nu}^{s}= & -\mathcal{A}^{\prime} \int d z \int d z^{\prime} z^{-1 / 2} z^{\prime-1 / 2} q^{\prime} K_{1}\left(q^{\prime} z^{\prime}\right) q K_{1}(q z) \\
& \times \bar{u}_{\sigma^{\prime}}\left(p^{\prime}\right)\left[J_{m R-1 / 2}\left(M z^{\prime}\right) P_{-}+J_{m R+1 / 2}\left(M z^{\prime}\right) P_{+}\right] \gamma_{\mu} \\
& \times G\left(z^{\prime}, z ; p+q\right) \frac{q_{\nu} q}{q^{2}}\left[J_{m R-1 / 2}(M z) P_{+}+J_{m R+1 / 2}(M z) P_{-}\right] u_{\sigma}(p) \\
& -i \mathcal{A}^{\prime} \bar{u}_{\sigma^{\prime}}\left(p^{\prime}\right) \gamma_{\mu} P_{+} u_{\sigma}(p) \frac{q_{\nu}}{q} \int d z z^{3} K_{1}(q z) K_{1}\left(q^{\prime} z\right) J_{m R-1 / 2}^{2}(M z) \\
& -i \mathcal{A}^{\prime} \bar{u}_{\sigma^{\prime}}\left(p^{\prime}\right) \gamma_{\mu} P_{-} u_{\sigma}(p) \frac{q_{\nu}}{q} \int d z z^{3} K_{1}(q z) K_{1}\left(q^{\prime} z\right) J_{m R+1 / 2}^{2}(M z), \\
T_{3 \mu \nu}^{s}= & -\mathcal{A}^{\prime} \int d z \int d z^{\prime} z^{-1 / 2} z^{\prime-1 / 2} q^{\prime} K_{1}\left(q^{\prime} z^{\prime}\right) q K_{1}(q z) \\
& \times \bar{u}_{\sigma^{\prime}}\left(p^{\prime}\right)\left[J_{m R-1 / 2}\left(M z^{\prime}\right) P_{-}+J_{m R+1 / 2}\left(M z^{\prime}\right) P_{+}\right] \frac{q_{\mu}^{\prime} q^{\prime \prime}}{q^{\prime 2}} \\
& \times G\left(z^{\prime}, z ; p+q\right) \gamma_{\nu}\left[J_{m R-1 / 2}(M z) P_{+}+J_{m R+1 / 2}(M z) P_{-}\right] u_{\sigma}(p) \\
& -i \mathcal{A}^{\prime} \bar{u}_{\sigma^{\prime}}\left(p^{\prime}\right) \gamma_{\nu} P_{+} u_{\sigma}(p) \frac{q_{\mu}^{\prime}}{q^{\prime}} \int d z z^{3} K_{1}(q z) K_{1}\left(q^{\prime} z\right) J_{m R-1 / 2}^{2}(M z) \\
& -i \mathcal{A}^{\prime} \bar{u}_{\sigma^{\prime}}\left(p^{\prime}\right) \gamma_{\nu} P_{-} u_{\sigma}(p) \frac{q_{\mu}^{\prime}}{q^{\prime}} \int d z z^{3} K_{1}(q z) K_{1}\left(q^{\prime} z\right) J_{m R+1 / 2}^{2}(M z)
\end{aligned}
$$

and

$$
\begin{aligned}
T_{4 \mu \nu}^{s}= & \mathcal{A}^{\prime} \int d z \int d z^{\prime} z^{-1 / 2} z^{\prime-1 / 2} q^{\prime} K_{1}\left(q^{\prime} z^{\prime}\right) q K_{1}(q z) \\
& \times \bar{u}_{\sigma^{\prime}}\left(p^{\prime}\right)\left[J_{m R-1 / 2}\left(M z^{\prime}\right) P_{-}+J_{m R+1 / 2}\left(M z^{\prime}\right) P_{+}\right] \frac{q_{\mu}^{\prime} q^{\prime}}{q^{\prime 2}} \\
& \times G\left(z^{\prime}, z ; p+q\right) \frac{q_{\nu} \phi}{q^{2}}\left[J_{m R-1 / 2}(M z) P_{+}+J_{m R+1 / 2}(M z) P_{-}\right] u_{\sigma}(p) \\
& +i \mathcal{A}^{\prime} \bar{u}_{\sigma^{\prime}}\left(p^{\prime}\right) q P_{+} u_{\sigma}(p) \frac{q_{\mu}^{\prime} q_{\nu}}{q^{\prime} q} \int d z z^{3} K_{1}\left(q^{\prime} z\right) K_{1}(q z) J_{m R-1 / 2}^{2}(M z) \\
& +i \mathcal{A}^{\prime} \bar{u}_{\sigma^{\prime}}\left(p^{\prime}\right) \not P_{-} u_{\sigma}(p) \frac{q_{\mu}^{\prime} q_{\nu}}{q^{\prime} q} \int d z z^{3} K_{1}\left(q^{\prime} z\right) K_{1}(q z) J_{m R+1 / 2}^{2}(M z) \\
& -\mathcal{A}^{\prime} \bar{u}_{\sigma^{\prime}}\left(p^{\prime}\right) \gamma^{5} u_{\sigma}(p) \frac{q_{\mu}^{\prime} q_{\nu}}{q^{\prime} q} \int d z z^{3} q^{\prime} K_{0}\left(q^{\prime} z\right) K_{1}(q z) J_{m R-1 / 2}(M z) J_{m R+1 / 2}(M z) .
\end{aligned}
$$


Using the same trick, it is then straightforward to calculate the $u$-channel graph as illustrated in Fig. (2) and obtain

$$
T_{\mu \nu}^{u}=T_{1 \mu \nu}^{u}+T_{2 \mu \nu}^{u}+T_{3 \mu \nu}^{u}+T_{4 \mu \nu}^{u}
$$

where

$$
\begin{aligned}
& T_{1 \mu \nu}^{u}=\mathcal{A}^{\prime} \int d z \int d z^{\prime} z^{-1 / 2} z^{\prime-1 / 2} q K_{1}\left(q z^{\prime}\right) q^{\prime} K_{1}\left(q^{\prime} z\right) \\
& \times \bar{u}_{\sigma^{\prime}}\left(p^{\prime}\right)\left[J_{m R-1 / 2}\left(M z^{\prime}\right) P_{-}+J_{m R+1 / 2}\left(M z^{\prime}\right) P_{+}\right] \gamma_{\nu} \\
& \times G\left(z^{\prime}, z ; p-q^{\prime}\right) \gamma_{\mu}\left[J_{m R-1 / 2}(M z) P_{+}+J_{m R+1 / 2}(M z) P_{-}\right] u_{\sigma}(p), \\
& T_{2 \mu \nu}^{u}=-\mathcal{A}^{\prime} \int d z \int d z^{\prime} z^{-1 / 2} z^{-1 / 2} q K_{1}\left(q z^{\prime}\right) q^{\prime} K_{1}\left(q^{\prime} z\right) \\
& \times \bar{u}_{\sigma^{\prime}}\left(p^{\prime}\right)\left[J_{m R-1 / 2}\left(M z^{\prime}\right) P_{-}+J_{m R+1 / 2}\left(M z^{\prime}\right) P_{+}\right] \gamma_{\nu} \\
& \times G\left(z^{\prime}, z ; p-q^{\prime}\right) \frac{q_{\mu}^{\prime} q^{\prime \prime}}{q^{\prime 2}}\left[J_{m R-1 / 2}(M z) P_{+}+J_{m R+1 / 2}(M z) P_{-}\right] u_{\sigma}(p) \\
& +i \mathcal{A}^{\prime} \bar{u}_{\sigma^{\prime}}\left(p^{\prime}\right) \gamma_{\nu} P_{+} u_{\sigma}(p) \frac{q_{\mu}^{\prime}}{q^{\prime}} \int d z z^{3} K_{1}(q z) K_{1}\left(q^{\prime} z\right) J_{m R-1 / 2}^{2}(M z) \\
& +i \mathcal{A}^{\prime} \bar{u}_{\sigma^{\prime}}\left(p^{\prime}\right) \gamma_{\nu} P_{-} u_{\sigma}(p) \frac{q_{\mu}^{\prime}}{q^{\prime}} \int d z z^{3} K_{1}(q z) K_{1}\left(q^{\prime} z\right) J_{m R+1 / 2}^{2}(M z), \\
& T_{3 \mu \nu}^{u}=-\mathcal{A}^{\prime} \int d z \int d z^{\prime} z^{-1 / 2} z^{\prime-1 / 2} q K_{1}\left(q z^{\prime}\right) q^{\prime} K_{1}\left(q^{\prime} z\right) \\
& \times \bar{u}_{\sigma^{\prime}}\left(p^{\prime}\right)\left[J_{m R-1 / 2}\left(M z^{\prime}\right) P_{-}+J_{m R+1 / 2}\left(M z^{\prime}\right) P_{+}\right] \frac{q_{\nu} q}{q^{2}} \\
& \times G\left(z^{\prime}, z ; p-q^{\prime}\right) \gamma_{\mu}\left[J_{m R-1 / 2}(M z) P_{+}+J_{m R+1 / 2}(M z) P_{-}\right] u_{\sigma}(p) \\
& +i \mathcal{A}^{\prime} \bar{u}_{\sigma^{\prime}}\left(p^{\prime}\right) \gamma_{\mu} P_{+} u_{\sigma}(p) \frac{q_{\nu}}{q} \int d z z^{3} K_{1}(q z) K_{1}\left(q^{\prime} z\right) J_{m R-1 / 2}^{2}(M z) \\
& +i \mathcal{A}^{\prime} \bar{u}_{\sigma^{\prime}}\left(p^{\prime}\right) \gamma_{\mu} P_{-} u_{\sigma}(p) \frac{q_{\nu}}{q} \int d z z^{3} K_{1}(q z) K_{1}\left(q^{\prime} z\right) J_{m R+1 / 2}^{2}(M z)
\end{aligned}
$$

and

$$
\begin{aligned}
T_{4 \mu \nu}^{u}= & \mathcal{A}^{\prime} \int d z \int d z^{\prime} z^{-1 / 2} z^{\prime-1 / 2} q K_{1}\left(q z^{\prime}\right) q^{\prime} K_{1}\left(q^{\prime} z\right) \\
& \times \bar{u}_{\sigma^{\prime}}\left(p^{\prime}\right)\left[J_{m R-1 / 2}\left(M z^{\prime}\right) P_{-}+J_{m R+1 / 2}\left(M z^{\prime}\right) P_{+}\right] \frac{q_{\nu} \phi}{q^{2}} \\
& \times G\left(z^{\prime}, z ; p-q^{\prime}\right) \frac{q_{\mu}^{\prime} q^{\prime \prime}}{q^{\prime 2}}\left[J_{m R-1 / 2}(M z) P_{+}+J_{m R+1 / 2}(M z) P_{-}\right] u_{\sigma}(p) \\
& -i \mathcal{A}^{\prime} \bar{u}_{\sigma^{\prime}}\left(p^{\prime}\right) q^{\prime} P_{+} u_{\sigma}(p) \frac{q_{\mu}^{\prime} q_{\nu}}{q^{\prime} q} \int d z z^{3} K_{1}\left(q^{\prime} z\right) K_{1}(q z) J_{m R-1 / 2}^{2}(M z)
\end{aligned}
$$




$$
\begin{aligned}
& -i \mathcal{A}^{\prime} \bar{u}_{\sigma^{\prime}}\left(p^{\prime}\right) q^{\prime \prime} P_{-} u_{\sigma}(p) \frac{q_{\mu}^{\prime} q_{\nu}}{q^{\prime} q} \int d z z^{3} K_{1}\left(q^{\prime} z\right) K_{1}(q z) J_{m R+1 / 2}^{2}(M z) \\
& -\mathcal{A}^{\prime} \bar{u}_{\sigma^{\prime}}\left(p^{\prime}\right) \gamma^{5} u_{\sigma}(p) \frac{q_{\mu}^{\prime} q_{\nu}}{q^{\prime}} \int d z z^{3} K_{0}(q z) K_{1}\left(q^{\prime} z\right) J_{m R-1 / 2}(M z) J_{m R+1 / 2}(M z)
\end{aligned}
$$

After some more algebra, one can find the total contribution is given by

$$
\begin{aligned}
T_{\mu \nu}= & T_{\mu \nu}^{s}+T_{\mu \nu}^{u} \\
= & \mathcal{A}^{\prime} \int d z \int d z^{\prime} z^{-1 / 2} z^{-1 / 2} q^{\prime} K_{1}\left(q^{\prime} z^{\prime}\right) q K_{1}(q z) \\
& \times \bar{u}_{\sigma^{\prime}}\left(p^{\prime}\right)\left[J_{m R-1 / 2}\left(M z^{\prime}\right) P_{-}+J_{m R+1 / 2}\left(M z^{\prime}\right) P_{+}\right]\left(\gamma_{\mu}-\frac{q_{\mu}^{\prime} q^{\prime}}{q^{\prime 2}}\right) \\
& \times G\left(z^{\prime}, z ; p+q\right)\left(\gamma_{\nu}-\frac{q_{\nu} \not}{q^{2}}\right)\left[J_{m R-1 / 2}(M z) P_{+}+J_{m R+1 / 2}(M z) P_{-}\right] u_{\sigma}(p) \\
& +\mathcal{A}^{\prime} \int d z \int d z^{\prime} z^{-1 / 2} z^{\prime-1 / 2} q K_{1}\left(q z^{\prime}\right) q^{\prime} K_{1}\left(q^{\prime} z\right) \\
& \times \bar{u}_{\sigma^{\prime}}\left(p^{\prime}\right)\left[J_{m R-1 / 2}\left(M z^{\prime}\right) P_{-}+J_{m R+1 / 2}\left(M z^{\prime}\right) P_{+}\right]\left(\gamma_{\nu}-\frac{q_{\nu} \not q}{q^{2}}\right) \\
& \times G\left(z^{\prime}, z ; p-q^{\prime}\right)\left(\gamma_{\mu}-\frac{q_{\mu}^{\prime} q^{\prime}}{q^{\prime 2}}\right)\left[J_{m R-1 / 2}(M z) P_{+}+J_{m R+1 / 2}(M z) P_{-}\right] u_{\sigma}(p) .(10)
\end{aligned}
$$

It is obvious that the current conservation, namely, $T_{\mu \nu} q^{\nu}=0$ and $q^{\prime \mu} T_{\mu \nu}=0$, is satisfied as a result of cancellation between $s$-channel and $u$-channel amplitudes due to gauge invariance. In addition, Eq. (103) can be recast into a simple form

$$
\begin{aligned}
T_{\mu \nu}= & -\mathcal{A}^{\prime} e_{\mu \alpha}^{L}\left(q^{\prime}\right) e_{\nu \beta}^{L}(q) \int_{0}^{\infty} d \omega \frac{\omega}{\omega^{2}+(p+q)^{2}-i \epsilon} \bar{u}_{\sigma^{\prime}}\left(p^{\prime}\right)\left[\mathcal{C}_{1}\left(q^{\prime}, \omega\right) P_{-}+\mathcal{C}_{2}\left(q^{\prime}, \omega\right) P_{+}\right] \\
& \times \gamma^{\alpha}[i(\not p+\not q)+\omega] \gamma^{\beta}\left[\mathcal{C}_{1}(q, \omega) P_{+}+\mathcal{C}_{2}(q, \omega) P_{-}\right] u_{\sigma}(p) \\
& -\mathcal{A}^{\prime} e_{\mu \beta}^{L}\left(q^{\prime}\right) e_{\nu \alpha}^{L}(q) \int_{0}^{\infty} d \omega \frac{\omega}{\omega^{2}+\left(p-q^{\prime}\right)^{2}-i \epsilon} \bar{u}_{\sigma^{\prime}}\left(p^{\prime}\right)\left[\mathcal{C}_{1}(q, \omega) P_{-}+\mathcal{C}_{2}(q, \omega) P_{+}\right] \\
& \times \gamma^{\alpha}\left[i\left(\not p-q^{\prime}\right)+\omega\right] \gamma^{\beta}\left[\mathcal{C}_{1}\left(q^{\prime}, \omega\right) P_{+}+\mathcal{C}_{2}\left(q^{\prime}, \omega\right) P_{-}\right] u_{\sigma}(p)
\end{aligned}
$$

where

$$
\begin{aligned}
e_{\mu \alpha}^{L}(q) & =g_{\mu \alpha}-\frac{q_{\mu} q_{\alpha}}{q^{2}} \\
\mathcal{C}_{1}(q, \omega) & =\int_{0}^{z_{0}} d z z^{2} q K_{1}(q z) J_{m R-1 / 2}(M z) J_{m R-1 / 2}(\omega z) \\
\mathcal{C}_{2}(q, \omega) & =\int_{0}^{z_{0}} d z z^{2} q K_{1}(q z) J_{m R+1 / 2}(M z) J_{m R+1 / 2}(\omega z) .
\end{aligned}
$$




\section{Real Compton Scattering}

In the case of real Compton scattering, both initial and final state photons are real. In the limit $q^{2}=0$, choosing the boundary condition $J_{\tau-2}\left(M z_{0}\right)=0$ with $\tau=m R+3 / 2$, we can have

$$
\begin{aligned}
& \mathcal{C}_{1}(0, \omega)=\frac{M}{M^{2}-\omega^{2}} z_{0} J_{\tau-2}\left(\omega z_{0}\right) J_{\tau-1}\left(M z_{0}\right) \\
& \mathcal{C}_{2}(0, \omega)=\frac{\omega}{M^{2}-\omega^{2}} z_{0} J_{\tau-2}\left(\omega z_{0}\right) J_{\tau-1}\left(M z_{0}\right)
\end{aligned}
$$

Here we would like to work in a limit that $M \rightarrow 0$ and $z_{0} \rightarrow \infty$ but with $M z_{0}$ fixed. If we take the limit $z_{0} \rightarrow \infty$, we can obtain

$$
\mathcal{C}_{1}(0, \omega) \rightarrow \frac{1}{M} \delta(\omega-M) \text { and } \mathcal{C}_{2}(0, \omega) \rightarrow \frac{1}{M} \delta(\omega-M)
$$

Setting both $q^{2}=0$ and $q^{2}=0$, the real Compton scattering amplitude is found to be

$$
\begin{aligned}
T_{\mu \nu}= & -\mathcal{A}^{\prime} z_{0}^{2} J_{\tau-1}^{2}\left(M z_{0}\right) \bar{u}_{\sigma^{\prime}}\left(p^{\prime}\right) \gamma_{\mu} \frac{i(p+\not q)+M}{M^{2}+(p+q)^{2}} \gamma_{\nu} u_{\sigma}(p) \\
& -\mathcal{A}^{\prime} z_{0}^{2} J_{\tau-1}^{2}\left(M z_{0}\right) \bar{u}_{\sigma^{\prime}}\left(p^{\prime}\right) \gamma_{\nu} \frac{i\left(p-q^{\prime \prime}\right)+M}{M^{2}+\left(p-q^{\prime}\right)^{2}} \gamma_{\mu} u_{\sigma}(p) .
\end{aligned}
$$

In such approximation, the real Compton scattering amplitude found above is parametrically the same as the Compton scattering amplitude of the fundamental particle of spin $1 / 2$.

\section{Deeply virtual Compton scattering}

In the case of DVCS, the incoming photon is virtual, and the outgoing photon is assumed to be real. Therefore, we set $q^{2}=0$ while $q^{2} \gg M^{2}$, and still let $z_{0} \rightarrow \infty$ but with $M z_{0}$ kept fixed. Thus, the scattering amplitude in Eq. (104) is found to be

$$
\begin{aligned}
T_{\mu \nu}= & -\mathcal{A}^{\prime} e_{\nu \beta}^{L}(q) \bar{u}_{\sigma^{\prime}}\left(p^{\prime}\right) \gamma_{\mu} \frac{i(p+\not q)+M}{M^{2}+(p+q)^{2}} \gamma^{\beta}\left[\mathcal{C}_{1}(q, M) P_{+}+\mathcal{C}_{2}(q, M) P_{-}\right] u_{\sigma}(p) \\
& -\mathcal{A}^{\prime} e_{\nu \alpha}^{L}(q) \bar{u}_{\sigma^{\prime}}\left(p^{\prime}\right)\left[\mathcal{C}_{1}(q, M) P_{-}+\mathcal{C}_{2}(q, M) P_{+}\right] \gamma^{\alpha} \frac{i\left(p-q^{\prime}\right)+M}{M^{2}+\left(p-q^{\prime}\right)^{2}} \gamma_{\mu} u_{\sigma}(p),
\end{aligned}
$$

where

$$
\begin{aligned}
& \mathcal{C}_{1}(q, M) \simeq \frac{2(\tau-1)}{M^{2}}\left(\frac{M^{2}}{q^{2}}\right)^{\tau-1}{ }_{2} F_{1}\left(\tau-\frac{3}{2}, \tau, 2 \tau-3,-\frac{4 M^{2}}{q^{2}}\right) \\
& \mathcal{C}_{2}(q, M) \simeq \frac{2 \tau}{M^{2}}\left(\frac{M^{2}}{q^{2}}\right)^{\tau}{ }_{2} F_{1}\left(\tau-\frac{1}{2}, \tau+1,2 \tau-1,-\frac{4 M^{2}}{q^{2}}\right) .
\end{aligned}
$$


To calculate the DVCS cross section, we define $T_{\mu \nu}=(2 \pi)^{4} \delta^{(4)}\left(p+q-p^{\prime}-q^{\prime}\right) \mathcal{M}_{\mu \nu}$. The amplitude $\mathcal{M}_{\mu \nu}$ can be written as

$$
\begin{aligned}
\mathcal{M}_{\mu \nu}= & -\frac{\mathcal{Q}^{2} c_{i}^{\prime} c_{f}^{\prime}}{z_{0}^{2}} e_{\nu \beta}^{L}(q) \bar{u}_{\sigma^{\prime}}\left(p^{\prime}\right) \gamma_{\mu} \frac{i(\not p+\not 1)+M}{M^{2}+(p+q)^{2}} \gamma^{\beta}\left[\mathcal{C}_{1}(q, M) P_{+}+\mathcal{C}_{2}(q, M) P_{-}\right] u_{\sigma}(p) \\
& -\frac{\mathcal{Q}^{2} c_{i}^{\prime} c_{f}^{\prime}}{z_{0}^{2}} e_{\nu \alpha}^{L}(q) \bar{u}_{\sigma^{\prime}}\left(p^{\prime}\right)\left[\mathcal{C}_{1}(q, M) P_{-}+\mathcal{C}_{2}(q, M) P_{+}\right] \gamma^{\alpha} \frac{i\left(p-q^{\prime}\right)+M}{M^{2}+\left(p-q^{\prime}\right)^{2}} \gamma_{\mu} u_{\sigma}(p)
\end{aligned}
$$

where one can see $\mathcal{M}_{\mu \nu}$ is dimensionless with our normalization. One can further simplify the amplitude as

$$
\begin{aligned}
\mathcal{M}_{\mu \nu}= & \mathcal{D}_{1}\left[\bar{u}_{\sigma^{\prime}}\left(p^{\prime}\right) \gamma_{\mu} \frac{i(\not p+\not q)+M}{M^{2}+(p+q)^{2}} \gamma_{\nu} u_{\sigma}(p)+\bar{u}_{\sigma^{\prime}}\left(p^{\prime}\right) \gamma_{\nu} \frac{i\left(\not p-q^{\prime}\right)+M}{M^{2}+\left(p+q^{\prime}\right)^{2}} \gamma_{\mu} u_{\sigma}(p)\right] \\
& +\mathcal{D}_{2} e_{\nu \alpha}^{L}(q) \bar{u}_{\sigma^{\prime}}\left(p^{\prime}\right) \gamma_{\mu} \frac{i(\not p+\not q)+M}{M^{2}+(p+q)^{2}} \gamma^{\alpha} \gamma^{5} u_{\sigma}(p) \\
& -\mathcal{D}_{2} e_{\nu \alpha}^{L}(q) \bar{u}_{\sigma^{\prime}}\left(p^{\prime}\right) \gamma^{5} \gamma^{\alpha} \frac{i\left(p-q^{\prime}\right)+M}{M^{2}+\left(p-q^{\prime}\right)^{2}} \gamma_{\mu} u_{\sigma}(p),
\end{aligned}
$$

with $\mathcal{D}_{1}=-\frac{\mathcal{Q}^{2} c_{i}^{\prime} c_{f}^{\prime}}{z_{0}^{2}} \frac{\mathcal{C}_{1}(q, M)+\mathcal{C}_{2}(q, M)}{2}$ and $\mathcal{D}_{2}=-\frac{\mathcal{Q}^{2} c_{i}^{\prime} c_{f}^{\prime}}{z_{0}^{2}} \frac{\mathcal{C}_{1}(q, M)-\mathcal{C}_{2}(q, M)}{2}$.

\section{Polarizations of photons}

Before we evaluate the DVCS cross section, we need to write down the polarization of real and virtual photons in the lab frame. First for a real photon, we suppose the momentum and the polarization of the photon can be written as

$$
\begin{aligned}
q_{\mu}^{\prime} & =\left(\omega^{\prime}, \omega^{\prime} \sin \theta, 0, \omega^{\prime} \cos \theta\right), \\
\varepsilon^{\prime \mu}\left(q^{\prime},+1\right) & =(0, \cos \theta, 0,-\sin \theta), \\
\varepsilon^{\prime \mu}\left(q^{\prime},-1\right) & =(0,0,1,0) .
\end{aligned}
$$

According to the current conservation

$$
\eta^{\mu \nu} q_{\mu}^{\prime} \mathcal{M}_{\nu}=0
$$

one obtains

$$
-\omega^{\prime} \mathcal{M}_{0}+\omega^{\prime} \mathcal{M}_{1} \sin \theta+\omega^{\prime} \mathcal{M}_{3} \cos \theta=0
$$

It is straightforward to show that

$$
\eta^{\mu \nu} \mathcal{M}_{\mu}^{*} \mathcal{M}_{\nu}=\left|\mathcal{M}_{1}\right|^{2}+\left|\mathcal{M}_{2}\right|^{2}+\left|\mathcal{M}_{3}\right|^{2}-\left|\mathcal{M}_{0}\right|^{2}=\sum_{\lambda} \epsilon^{* \mu} \epsilon^{\nu} \mathcal{M}_{\mu}^{*} \mathcal{M}_{\nu}
$$


Therefore, we see that $\sum_{\lambda} \epsilon^{* \mu} \epsilon^{\nu}=\eta^{\mu \nu}$.

Now for a virtual photon, we find that the momentum and the polarization are

$$
\begin{aligned}
q_{\mu} & =\left(q_{0}, 0,0, q_{3}\right), \\
\varepsilon^{\mu}(q, 0) & =\frac{1}{q}\left(q_{3}, 0,0, q_{0}\right), \\
\varepsilon^{\mu}(q,+1) & =(0,1,0,0), \\
\varepsilon^{\mu}(q,-1) & =(0,0,1,0),
\end{aligned}
$$

where $q^{2}=q_{3}^{2}-q_{0}^{2}$. According to the current conservation

$$
\eta^{\mu \nu} q_{\mu} \mathcal{M}_{\nu}=0
$$

one gets

$$
-q_{0} \mathcal{M}_{0}+q_{3} \mathcal{M}_{3}=0 .
$$

It is easy to see that

$$
\begin{aligned}
\eta^{\mu \nu} \mathcal{M}_{\mu}^{*} \mathcal{M}_{\nu} & =\left|\mathcal{M}_{1}\right|^{2}+\left|\mathcal{M}_{2}\right|^{2}+\left|\mathcal{M}_{3}\right|^{2}-\left|\mathcal{M}_{0}\right|^{2} \\
& =\left|\mathcal{M}_{1}\right|^{2}+\left|\mathcal{M}_{2}\right|^{2}-\frac{q^{2}}{q_{0}^{2}}\left|\mathcal{M}_{3}\right|^{2},
\end{aligned}
$$

and

$$
\begin{aligned}
\sum_{\lambda} \epsilon^{* \mu} \epsilon^{\nu} \mathcal{M}_{\mu}^{*} \mathcal{M}_{\nu} & =\left|\mathcal{M}_{1}\right|^{2}+\left|\mathcal{M}_{2}\right|^{2}+\left(-\frac{q_{3}}{q} \mathcal{M}_{0}^{*}+\frac{q_{0}}{q} \mathcal{M}_{3}^{*}\right)\left(-\frac{q_{3}}{q} \mathcal{M}_{0}+\frac{q_{0}}{q} \mathcal{M}_{3}\right) \\
& =\left|\mathcal{M}_{1}\right|^{2}+\left|\mathcal{M}_{2}\right|^{2}+\frac{q^{2}}{q_{0}^{2}}\left|\mathcal{M}_{3}\right|^{2} .
\end{aligned}
$$

Therefore, for a virtual photon, we know

$$
\sum_{\lambda} \epsilon^{* \mu} \epsilon^{\nu} \neq \eta^{\mu \nu} \text {, but } \sum_{\lambda}(-1)^{\lambda+1} \epsilon^{* \mu} \epsilon^{\nu}=\eta^{\mu \nu} .
$$

2. DVCS cross sections

Using the results from the last subsection, we can find the following identity

$$
\begin{aligned}
2\left(\sigma_{T}+\sigma_{L}\right) & =\left|\mathcal{M}_{1}\right|^{2}+\left|\mathcal{M}_{2}\right|^{2}+2 \frac{q^{2}}{q_{0}^{2}}\left|\mathcal{M}_{3}\right|^{2}, \\
& =\eta^{\mu \nu} \mathcal{M}_{\mu}^{*} \mathcal{M}_{\nu}+3 \frac{q^{2}}{q_{3}^{2} M^{2}} p^{\mu} p^{\nu} \mathcal{M}_{\mu}^{*} \mathcal{M}_{\nu},
\end{aligned}
$$


Together with the definition

$$
W_{\nu \nu^{\prime}}=\varepsilon^{\mu}\left(q^{\prime}\right) \varepsilon^{* \mu^{\prime}}\left(q^{\prime}\right) \mathcal{M}_{\mu \nu} \mathcal{M}_{\mu^{\prime} \nu^{\prime}}^{*}
$$

after some tedious but straightforward computation, we find

$$
\sum_{\lambda} \epsilon^{* \mu} \epsilon^{\nu} W_{\mu \nu}=8 \mathcal{D}_{1} \mathcal{D}_{1}\left\{A_{s s}^{(1)}+A_{u u}^{(1)}+A_{u s}^{(1)}\right\}+8 \mathcal{D}_{2} \mathcal{D}_{2}\left\{A_{s s}^{(2)}+A_{u u}^{(2)}+A_{u s}^{(2)}\right\}
$$

with

$$
\begin{aligned}
A_{s s}^{(1)}= & \frac{1}{\tilde{s}^{2}}\left(4 M^{4}-4 M^{2} \tilde{s}-2 M^{2} t-2 M^{2} \tilde{u}-\tilde{s} \tilde{u}\right) \\
& +\frac{3 q^{2}}{q_{3}^{2} \tilde{s}^{2}}\left(M^{2} t+4 M^{4}-M^{2} \tilde{s}+M^{2} \tilde{u}+\frac{1}{2} \tilde{u} \tilde{s}\right) \\
A_{u u}^{(1)}= & \frac{1}{\tilde{u}^{2}}\left(4 M^{4}-2 M^{2} \tilde{s}-2 M^{2} t-4 M^{2} \tilde{u}-\tilde{s} \tilde{u}\right) \\
& +\frac{3 q^{2}}{q_{3}^{2} \tilde{u}^{2}}\left[\left(t+2 M^{2}\right)\left(M^{2}-\tilde{u}-\frac{\tilde{u}^{2}}{2 M^{2}}\right)+2 M^{4}-\frac{1}{2} \tilde{u} \tilde{s}-M^{2}(\tilde{s}+\tilde{u})\right] \\
A_{u s}^{(1)}= & \frac{2}{\tilde{u} \tilde{s}}\left[M^{2}\left(4 M^{2}-\tilde{s}-\tilde{u}\right)-t(t+\tilde{s}+\tilde{u})\right] \\
& +\frac{3 q^{2}}{q_{3}^{2} \tilde{u} \tilde{s}}\left[\left(t+2 M^{2}\right)\left(4 M^{2}+\tilde{u}+t\right)-\tilde{u}^{2}+2 M^{2} \tilde{s}\right],
\end{aligned}
$$

and

$$
\begin{aligned}
& A_{s s}^{(1)}+A_{u u}^{(1)}+A_{u s}^{(1)} \\
= & -\frac{1}{\tilde{s}^{2} \tilde{u}^{2}}\left\{2 \tilde{s} \tilde{u}\left[t(t+\tilde{s}+\tilde{u})-M^{2}\left(4 M^{2}-\tilde{s}-\tilde{u}\right)\right]\right. \\
& -\tilde{u}^{2}\left(4 M^{4}-4 M^{2} \tilde{s}-2 M^{2} t-2 M^{2} \tilde{u}-\tilde{s} \tilde{u}\right) \\
& \left.-\tilde{s}^{2}\left(4 M^{4}-4 M^{2} \tilde{u}-2 M^{2} t-2 M^{2} \tilde{s}-\tilde{s} \tilde{u}\right)\right\} \\
& +\frac{3 q^{2}}{q_{3}^{2} \tilde{s}^{2} \tilde{u}^{2}}\left(4 M^{2}-\tilde{s}+t+\tilde{u}-\frac{\tilde{s} \tilde{u}}{2 M^{2}}\right)\left[M^{2}(\tilde{s}+\tilde{u})^{2}+t \tilde{s} \tilde{u}\right] \\
= & -\frac{1}{\tilde{s}^{2} \tilde{u}^{2}}\left\{\left(\tilde{s}^{2}+\tilde{u}^{2}\right) \tilde{s} \tilde{u}+2\left(q^{2}-2 M^{2}\right)\left[M^{2}(\tilde{s}+\tilde{u})^{2}+\tilde{u} \tilde{s} t\right]\right\} \\
& +\frac{3 q^{2}}{q_{3}^{2} \tilde{s}^{2} \tilde{u}^{2}}\left(4 M^{2}-\tilde{s}+t+\tilde{u}-\frac{\tilde{s} \tilde{u}}{2 M^{2}}\right)\left[M^{2}(\tilde{s}+\tilde{u})^{2}+t \tilde{s} \tilde{u}\right],
\end{aligned}
$$

and

$$
\begin{aligned}
A_{s s}^{(2)}= & -\frac{1}{2 q^{2} \tilde{s}^{2}}\left[2 M^{2}\left(8 M^{2} q^{2}+2 q^{4}-4 q^{2} \tilde{s}-\tilde{s}^{2}\right)-\tilde{s}^{2}(\tilde{s}+\tilde{u})+q^{2} \tilde{s}(\tilde{s}+2 \tilde{u})\right] \\
& -\frac{3}{8 q^{2} q_{3}^{2}}\left[4 M^{2} q^{2}+\left(q^{2}-\tilde{s}\right)^{2}\right]\left(-2+\frac{t}{M^{2}}\right)
\end{aligned}
$$




$$
\begin{aligned}
& A_{u u}^{(2)}=-\frac{1}{2 q^{2} \tilde{u}^{2}}\left[2 M^{2}\left(8 M^{2} q^{2}+2 q^{4}-4 q^{2} \tilde{u}-\tilde{u}^{2}\right)-\tilde{u}^{2}(\tilde{s}+\tilde{u})+q^{2} \tilde{u}(\tilde{u}+2 \tilde{s})\right] \\
&-\frac{3}{8 q^{2} q^{2} \tilde{u}^{2}}\left[2\left(4 M^{2} q^{2} \tilde{s}^{2}+8 M^{2} q^{2} \tilde{s} \tilde{u}+4 q^{4} \tilde{s} \tilde{u}+q^{4} \tilde{u}^{2}-2 q^{2} \tilde{s}^{2} \tilde{u}-\tilde{s}^{2} \tilde{u}^{2}\right)\right. \\
&\left.\quad+\frac{t}{M^{2}}\left(q^{2}+\tilde{s}\right)^{2} \tilde{u}^{2}\right] \\
& A_{u s}^{(2)}=-\frac{1}{q^{2} \tilde{u} \tilde{s}}\left[4 M^{2} q^{2}\left(4 M^{2}+2 q^{2}+t\right)-2 M^{2}\left(\tilde{s}^{2}+\tilde{u}^{2}+\tilde{s} \tilde{u}\right)+\left(2 q^{4}-\tilde{s} \tilde{u}\right) t\right] \\
& \quad-\frac{3}{4 q^{2} q_{3}^{2} \tilde{u}}\left[2\left(4 M^{2} q^{2} \tilde{u}+3 q^{4} \tilde{u}+q^{2} \tilde{s}^{2}-2 q^{2} \tilde{s} \tilde{u}-q^{2} \tilde{u}^{2}-\tilde{s}^{3}-\tilde{s}^{2} \tilde{u}-\tilde{s} \tilde{u}^{2}\right)\right. \\
&\left.\quad+\frac{t}{M^{2}}\left(q^{4}-\tilde{s}^{2}\right) \tilde{u}\right]
\end{aligned}
$$

and

$$
\begin{aligned}
& A_{s s}^{(2)}+A_{u u}^{(2)}+A_{u s}^{(2)} \\
= & -\frac{1}{q^{2} \tilde{s}^{2} \tilde{u}^{2}}\left[2 M^{2}(\tilde{s}+\tilde{u})^{2}\left(4 M^{2} q^{2}+q^{4}-\tilde{s} \tilde{u}\right)+q^{2} \tilde{s} \tilde{u}\left(\tilde{s}^{2}+\tilde{u}^{2}+8 M^{2} t+2 q^{2} t\right)\right] \\
& -\frac{3}{2 q^{2} q_{3}^{2} \tilde{u}^{2}}\left[\left(2 M^{2} q^{2}-\tilde{s} \tilde{u}\right)(\tilde{s}+\tilde{u})^{2}+2 q^{4} \tilde{u}(\tilde{s}+\tilde{u})+q^{2} \tilde{u}^{2} t\left(2+q^{2} / M^{2}\right)\right] .
\end{aligned}
$$

The Mandelstam variables used above are defined as follows

$$
\begin{aligned}
\tilde{s} & =s+M^{2}=\left(p^{\prime}+q^{\prime}\right)^{2}+M^{2}=2 p^{\prime} \cdot q^{\prime}, \\
\tilde{u} & =u+M^{2}=\left(p-q^{\prime}\right)^{2}+M^{2}=-2 p \cdot q^{\prime}, \\
t & =\left(p-p^{\prime}\right)^{2} .
\end{aligned}
$$

Using the lab frame we define in Eqs.(46) and Eq.(47), along with the phase space integral

$$
\int d \Pi_{2}==\frac{1}{8 \pi \sqrt{4 M^{2} q^{2}+\left(W^{2}+q^{2}-M^{2}\right)^{2}}} \int d t, \quad \text { with } \quad W^{2}=2 M q_{0}-q^{2}+M^{2},
$$

we can write cross-section formula as

$$
\begin{aligned}
\frac{d \sigma^{\mathrm{Un}}}{d t}= & \frac{1}{2 q_{0}} \frac{1}{2 M} \frac{1}{4 \pi} \frac{\omega^{\prime 2}}{2 M q_{0}-q^{2}} \frac{1}{4} 2\left(\sigma_{T}+\sigma_{L}\right) \\
= & \frac{1}{16 \pi\left(W^{2}+q^{2}-M^{2}\right) \sqrt{4 M^{2} q^{2}+\left(W^{2}+q^{2}-M^{2}\right)^{2}}} \\
& \times\left[2 \mathcal{D}_{1}^{2}\left(A_{s s}^{(1)}+A_{u u}^{(1)}+A_{u s}^{(1)}\right)+2 \mathcal{D}_{2}^{2}\left(A_{s s}^{(2)}+A_{u u}^{(2)}+A_{u s}^{(2)}\right)\right] .
\end{aligned}
$$

The above equation is one of our major results in this paper. Assuming the QCD coupling is large during the interaction due to confinement, we can compare this result to the HERA data. We plot the integrated cross section as a function of $Q^{2}$ in Fig. (4) As shown in Fig. 4, 


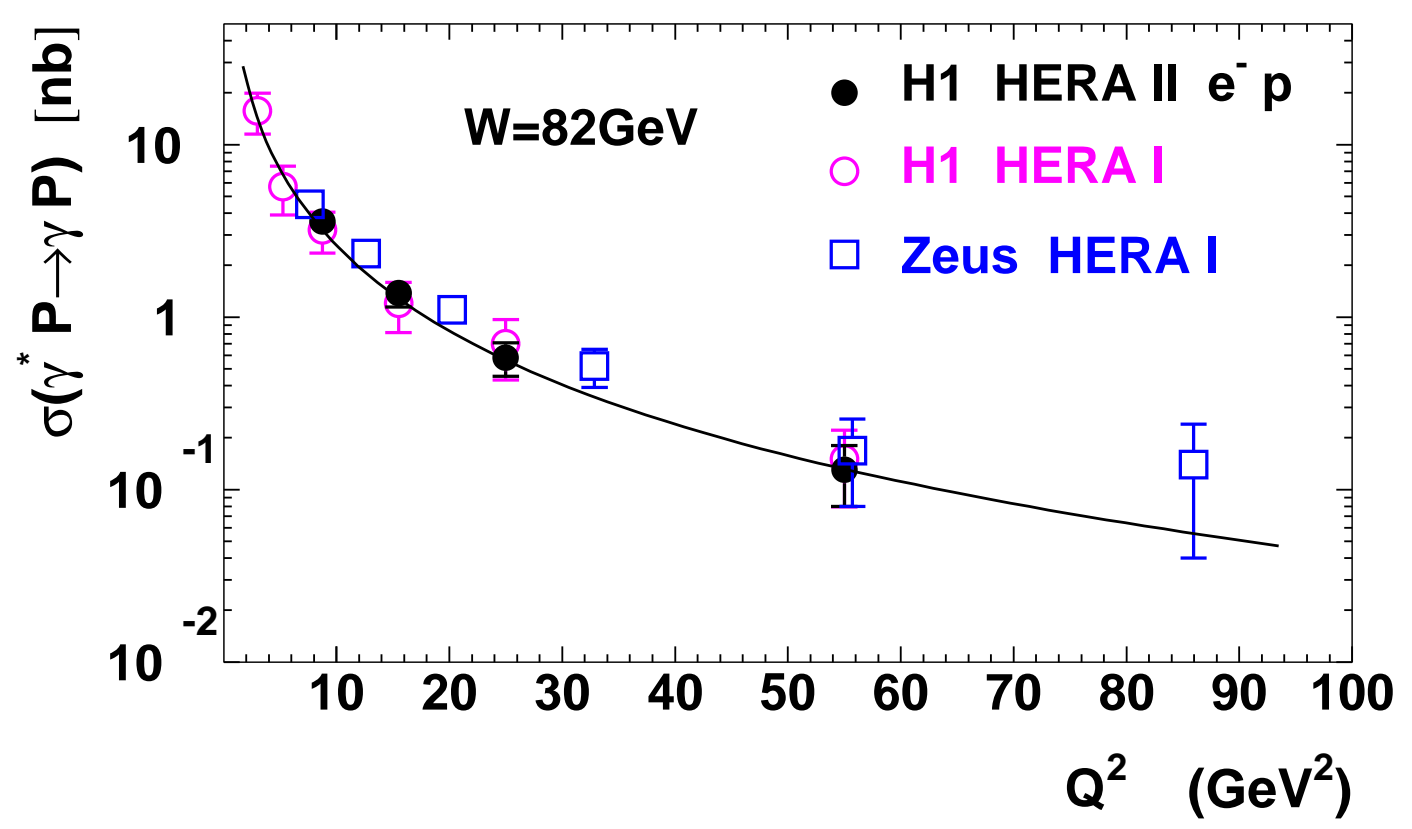

FIG. 4: The DVCS $\left(\gamma^{*} p \rightarrow \gamma p\right)$ cross section as a function of $Q^{2}$ for $W=82 \mathrm{GeV}$ and $|t|<1 \mathrm{GeV}^{2}$. The data points are taken from Refs. [46 48]. The solid line represents the dilatino DVCS cross section in Eq. (149) after integrating over $t$. We set $\tau=2$, the mass $M=0.938 \mathrm{GeV}$ to be the proton mass and $q^{2}=Q^{2}$. We have used one of the data points to fix the overall constant.

we compare the DVCS cross section in Eq. (149) after integrating over $t(|t|<1)$ to the low-energy H1 and the ZEUS data[46-48] and find that our result is consistent with the data. Equation (149) only includes the $s$-channel and $u$-channel graphs. There should be the $t$-channel graviton exchange contribution which is discussed in the next section in the plot. However, we argue that the $s$-channel and $u$-channel contributions are dominant in the low-energy region, whereas the $t$-channel graviton exchange dominates in the high-energy limit. In addition, technically, it is hard to fix the normalization of the $t$-channel graph as compared to the $s$-channel and $u$-channel graphs. It is obvious that the $s$-channel and $u$ channel contributions are proportional to $\mathcal{Q}^{2}$, with $\mathcal{Q}$ being the charge of the targets. On the other hand, the $t$-channel graviton exchange only couples to the energy momentum tensors of the target hadrons, and it does not depend on the quantum number of the target. In QCD, 


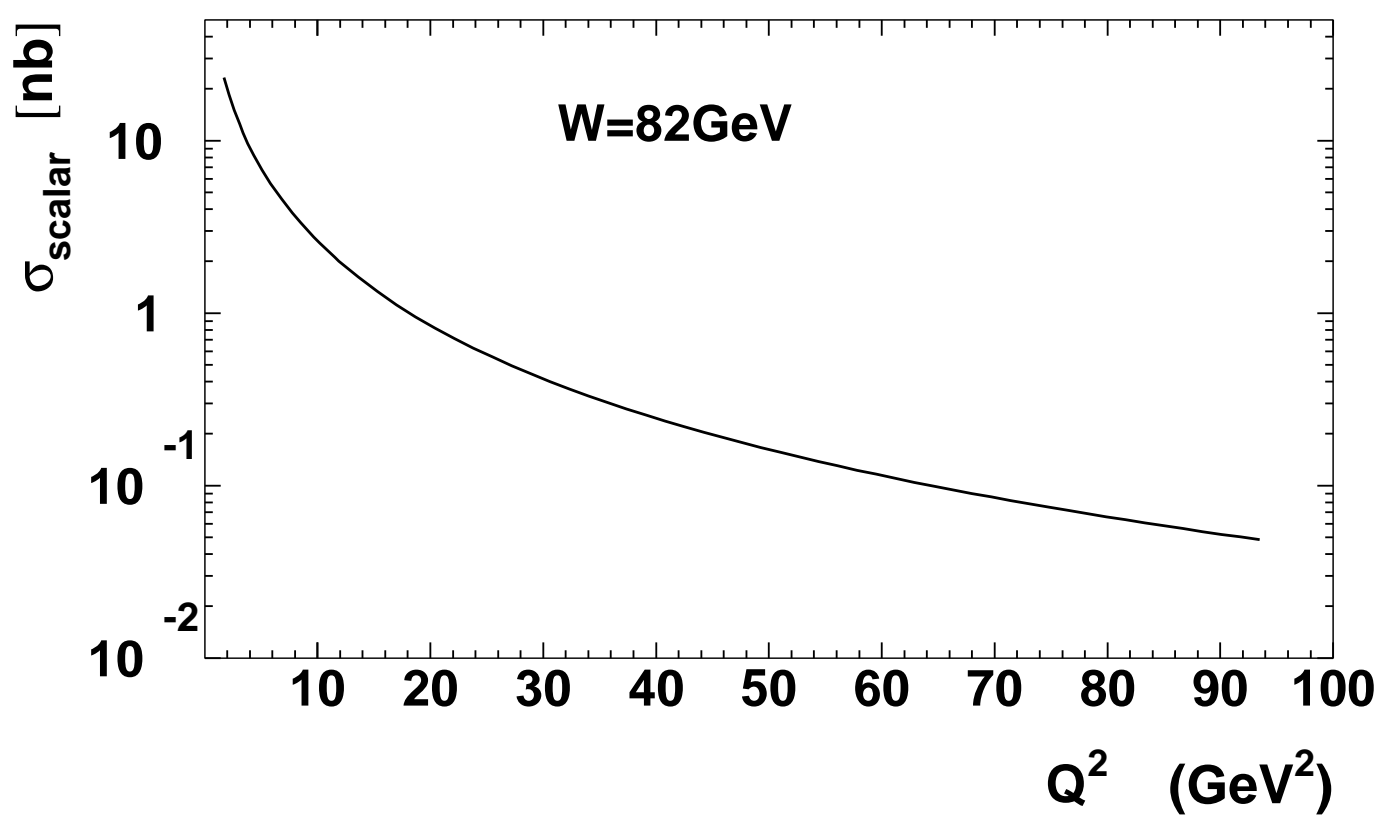

FIG. 5: The scalar DVCS cross section as a function of $Q^{2}$ for $W=82 G e V$ and $|t|<1 G e V^{2}$. The solid line represents the dilaton DVCS cross section calculated in Sec. II We set $\Delta=2$ and use the same normalization and parameters as the dilatino in the plot.

the situation is quite similar. In the low-energy region, where valence quark contribution dominates, the scattering amplitude certainly is proportional to $e^{2}$, where $e$ is the proton charge. However, in the high-energy region, where the Pomeron exchange starts to dominate the amplitude, we find the parton distribution becomes universal and independent of the quantum number of the target hadron. Therefore, assuming the $t$-channel graviton exchange graphs are small in the low-energy region, we neglect this contribution when we compare the calculated cross section to the low-energy H1 and the ZEUS data[46 48].

As a comparison, we also plot the integrated cross section for a scalar target, which is calculated in Sec. III, as function of $Q^{2}$ in Fig. 5. We use the same parameter and overall constant as in Fig. 4, Numerically, there is little difference between these two plots. 


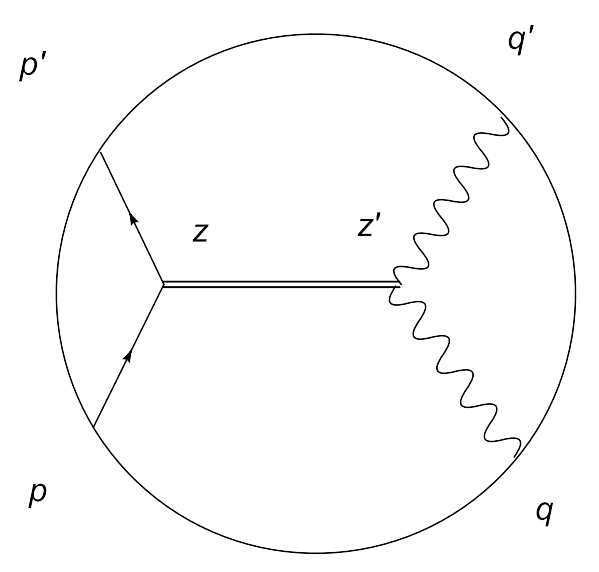

FIG. 6: $t$-channel graviton exchange

\section{T-CHANNEL GRAVITON EXCHANGES}

The exact evaluation of the $t$-channel graviton exchange graph as illustrated in Fig. 6 is not an easy task. Here we provide a heuristic derivation at the large- $s$ limit where the $t$-channel graviton exchange contribution dominates, with $s$ being the center of mass energy. Therefore, hereafter we neglect the mass terms in the energy momentum tensors and only keep the leading order terms. For simplicity, we also drop the $S^{5}$ dependence of the wave functions, because it only contributes an overall constant. It is well-known that the graviton couples to the energy momentum tensor. The $t$-channel graviton exchange contribution does not depend on the charge of the target hadron $(\mathcal{Q})$, which is uniquely different from the $s$ channel and $u$-channel graphs' contributions computed above. Technically, it is hard to normalize the $t$-channel graviton contribution as compared to the $s$-channel and $u$-channel graphs' contributions. We assume that the $t$-channel contribution is small at low energy. However, it is the dominant contribution at high energy due to its energy dependence. In this section, we set the curvature of the AdS space $R=1$, since the final results do not depend on $R$.

The $t$-channel graviton exchange amplitude between the dilaton target and the photon can be written in the following:

$$
\mathcal{A}^{G}=n^{\mu} T_{\mu \nu}^{G} n^{\prime * \nu}=\kappa^{2} \int d^{5} y d^{5} y^{\prime} T_{m n}^{\Phi}(y) G^{m n k l}\left(y, y^{\prime}\right) T_{k l}^{A}\left(y^{\prime}\right)
$$

where $n^{\mu}$ and $n^{\prime * \nu}$ are the polarization vectors and $\kappa$ is the gravitational coupling to the 
stress-energy tensor. $G^{m n k l}(x, y)$ is the graviton propagator which can be written as 49]

$$
G^{m n k l}\left(y, y^{\prime}\right)=\left(g^{m k} g^{n l}+g^{m l} g^{n k}-\eta g^{m n} g^{k l}\right) G\left(y, y^{\prime}\right)
$$

with $\eta=\frac{2}{D-2}$. Strictly speaking, one should use the full graviton propagator derived in $A d S_{5}$ space as in Ref. [40]. However, in the high-energy limit, one can still obtain the correct expression using the above graviton propagator (see, e.g., in Refs. [5, 41]). The stress-energy tensor $T_{m n}^{\Phi}$ for a massless dilaton field is

$$
T_{m n}^{\Phi}=\left(g_{m \alpha} g_{n \beta}+g_{m \beta} g_{n \alpha}-g_{m n} g_{\alpha \beta}\right) \partial^{\alpha} \Phi^{*} \partial^{\beta} \Phi
$$

We put $\Phi$ as the initial dilaton wave function and $\Phi^{*}$ as the final dilaton wave function. The stress-energy tensor $T_{k l}^{A}$ for a Kaluza-Klein gauge field can be written as

$$
T_{k l}^{A}=g^{\rho \sigma} F_{k \rho} F_{l \sigma}-\frac{1}{4} g_{k l} F_{\rho \sigma} F^{\rho \sigma},
$$

with

$$
\begin{aligned}
& F_{\mu \nu}=i\left(q_{\mu} n_{\nu}-q_{\nu} n_{\mu}\right) e^{i q \cdot y} q z K_{1}(q z), \\
& F_{\mu z}=\left(n_{\mu} q^{2}-q_{\mu} q \cdot n\right) e^{i q \cdot y} z K_{0}(q z) .
\end{aligned}
$$

The indices $\mu, \nu$ denote the four dimensions in Minkowski spacetime, and the rest of the indices $m, n, k, l, \cdots$ are defined in $\mathrm{AdS}_{5}$ space. It is easy to work out the tensor contractions and obtain

$$
\begin{aligned}
& T_{m n}^{\Phi}(y) G^{m n k l}\left(y, y^{\prime}\right) T_{k l}^{A}\left(y^{\prime}\right) \\
\propto & 2\left(\partial^{k} \Phi^{*} \partial^{l} \Phi+\partial^{k} \Phi \partial^{l} \Phi^{*}\right) F_{k \rho} F_{l \sigma} g^{\rho \sigma}-\partial^{k} \Phi \partial_{k} \Phi F_{\rho \sigma} F^{\rho \sigma}+\cdots
\end{aligned}
$$

This expression agrees with Ref. [5] in the forward limit. At high energy[41], the first term in the above effective action dominates, because it gives rise to terms like $p \cdot q$, which is large as compared to $q^{2}$ and $M^{2}$. Therefore, the graviton exchange amplitude for the Compton scattering can be written as

$$
\mathcal{A}^{G}=n^{\mu} \mathcal{T}_{\mu \nu}^{G} n^{\prime * \nu}=\int d^{5} y \sqrt{-g} d^{5} y^{\prime} \sqrt{-g^{\prime}} 2\left(\partial^{k} \Phi^{*} \partial^{l} \Phi+\partial^{k} \Phi \partial^{k} \Phi^{*}\right) g^{\rho \sigma} F_{k \rho} F_{l \sigma} G\left(y, y^{\prime}\right),
$$

It is straightforward to find that

$$
\begin{aligned}
& g^{\rho \sigma} F_{\mu \rho}(q) F_{\nu \sigma}\left(q^{\prime}\right) \\
= & \left(n_{\mu} q^{2}-q_{\mu} q \cdot n\right)\left(n_{\nu}^{\prime} q^{\prime 2}-q_{\nu}^{\prime} q^{\prime} \cdot n^{\prime}\right) \frac{z^{4}}{R^{2}} e^{i\left(q-q^{\prime}\right) \cdot y} K_{0}(q z) K_{0}\left(q^{\prime} z\right) \\
& +\left(n \cdot n^{\prime} q_{\mu} q_{\nu}^{\prime}-q \cdot n^{\prime} n_{\mu} q_{\nu}^{\prime}-q^{\prime} \cdot n q_{\mu} n_{\nu}^{\prime}+q \cdot q^{\prime} n_{\mu} n_{\nu}^{\prime}\right) \frac{z^{4}}{R^{2}} e^{i\left(q-q^{\prime}\right) \cdot y} q^{\prime} q K_{1}(q z) K_{1}\left(q^{\prime} z\right)(15)
\end{aligned}
$$


Following the derivation in Ref.[24], we get

$$
\begin{aligned}
\mathcal{T}_{\mu \nu}^{G} \propto & \mathcal{A}^{\prime \prime}\left[\left(p_{\mu}+\frac{q_{\mu}}{2 x}\right)\left(p_{\nu}^{\prime}+\frac{q_{\nu}^{\prime}}{2 x^{\prime}}\right)+\left(p_{\mu}^{\prime}-\frac{q_{\mu}}{2 \tilde{x}}\right)\left(p_{\nu}-\frac{q_{\nu}^{\prime}}{2 \tilde{x}^{\prime}}\right)\right] q^{2} q^{\prime 2} \mathcal{T}_{1}^{G} \\
+ & \mathcal{A}^{\prime \prime}\left[\begin{array}{c}
\frac{q^{\prime} q}{4 x x^{\prime}}\left(\eta_{\mu \nu}+\frac{2 x}{q^{2}} p_{\mu} q_{\nu}+\frac{2 x^{\prime}}{q^{\prime 2}} q_{\mu}^{\prime} p_{\nu}^{\prime}+\frac{4 x x^{\prime}}{q^{2} q^{\prime 2}} q \cdot q^{\prime} p_{\mu} p_{\nu}^{\prime}\right) \\
\left.+\frac{q^{\prime} q}{4 \tilde{x} \tilde{x}^{\prime}}\left(\eta_{\mu \nu}-\frac{2 \tilde{x}}{q^{2}} p_{\mu}^{\prime} q_{\nu}-\frac{2 \tilde{x}^{\prime}}{q^{\prime 2}} q_{\mu}^{\prime} p_{\nu}+\frac{4 \tilde{x} \tilde{x}^{\prime}}{q^{2} q^{\prime 2}} q \cdot q^{\prime} p_{\mu}^{\prime} p_{\nu}\right)\right] q^{2} q^{\prime 2} \mathcal{T}_{2}^{G}
\end{array}\right.
\end{aligned}
$$

with $\mathcal{A}^{\prime \prime} \propto(2 \pi)^{4} \delta\left(p+q-p^{\prime}-q^{\prime}\right), x=-\frac{q^{2}}{2 p \cdot q}, x^{\prime}=-\frac{q^{\prime 2}}{2 p^{\prime} \cdot q^{\prime}}, \tilde{x}=\frac{q^{2}}{2 p^{\prime} \cdot q}, \tilde{x}^{\prime}=\frac{q^{\prime 2}}{2 p \cdot q^{\prime}}$ and

$$
\begin{aligned}
\mathcal{T}_{1}^{G}= & \int d z z^{3} K_{0}(q z) K_{0}\left(q^{\prime} z\right) \int d z^{\prime} z^{\prime 3} J_{\Delta-2}^{2}\left(M z^{\prime}\right) \\
& \times \int_{\mathcal{C}} \frac{d j}{2 i} \frac{1+e^{-i \pi j}}{\sin \pi j} \frac{\left(\alpha^{\prime} s\right)^{j-2+\alpha^{\prime} t z^{\prime 2}}}{\sqrt{j-j_{0}}}\left(\frac{z^{2}}{z^{\prime 2}}\right)^{1-\gamma}, \\
\mathcal{T}_{2}^{G}= & \int d z z^{3} K_{1}(q z) K_{1}\left(q^{\prime} z\right) \int d z^{\prime} z^{\prime 3} J_{\Delta-2}^{2}\left(M z^{\prime}\right) \\
& \times \int_{\mathcal{C}} \frac{d j}{2 i} \frac{1+e^{-i \pi j}}{\sin \pi j} \frac{\left(\alpha^{\prime} s\right)^{j-2+\alpha^{\prime} t z^{\prime 2}}}{\sqrt{j-j_{0}}}\left(\frac{z^{2}}{z^{\prime 2}}\right)^{1-\gamma},
\end{aligned}
$$

with $\alpha^{\prime}=R^{2} / \sqrt{\lambda}$ and $\gamma \simeq 1-\frac{1}{\sqrt{\lambda}}$. Here we have neglected the contributions of $g^{\rho \sigma} F_{z \rho}(q) F_{\nu \sigma}\left(q^{\prime}\right)$ and $g^{\rho \sigma} F_{z \rho}(q) F_{z \sigma}\left(q^{\prime}\right)$ since they are subleading at the high-energy limit. The contour $\mathcal{C}$ runs parallel to the imaginary axis from $j-i \infty$ to $j+i \infty$ with $j_{0} \equiv 2-\frac{2}{\sqrt{\lambda}}<$ $j<2$. It is interesting to note that $\mathcal{T}_{\mu \nu}^{G}$ is always conserved, namely, $q^{\mu} \mathcal{T}_{\mu \nu}^{G}=0$ and $q^{\prime \nu} \mathcal{T}_{\mu \nu}^{G}=0$, thanks to its coupling to the photon energy momentum tensor and the equation of motion.

Assuming that the interaction is $\operatorname{local}^{5}$, one obtains

$$
\begin{aligned}
& \mathcal{T}_{1}^{G}=\int d z z^{3} K_{0}(q z) K_{0}\left(q^{\prime} z\right) z^{4} J_{\Delta-2}^{2}(M z), \\
& \mathcal{T}_{2}^{G}=\int d z z^{3} K_{1}(q z) K_{1}\left(q^{\prime} z\right) z^{4} J_{\Delta-2}^{2}(M z) .
\end{aligned}
$$

In the forward Compton scattering limit, our result agrees with the one found in Refs. [5, 24]. If the energy gets so high that $\frac{1}{\sqrt{\lambda}} \ln \alpha^{\prime} s$ becomes of order 1 , the imaginary part of the graviton exchange amplitude can no longer be assumed local due to diffusion [5, 24]. Because graviton has a spin of 2 , the forward scattering amplitude is proportional to $\frac{1}{x^{2}}$ up to some curvature corrections.

\footnotetext{
${ }^{5}$ Essentially, the $t$-channel graviton exchange is non-local. Here we adopt this local approximation to show that the imaginary part of this amplitude in the forward limit can smoothly match the DIS calculation 5 , 24.
} 
For a dilatino target, we use the following dilatino energy momentum tensor

$$
T_{\Psi}^{m n}=\frac{1}{2}\left(\bar{\Psi}^{*} \gamma^{m} \vec{\partial}^{n} \Psi+\bar{\Psi}^{*} \gamma^{m} \overleftarrow{\partial^{n}} \Psi+\bar{\Psi}^{*} \gamma^{n} \vec{\partial}^{m} \Psi+\bar{\Psi}^{*} \gamma^{n} \overleftarrow{\partial^{m}} \Psi\right)
$$

From the above calculation for the dilaton, one can easily see that the leading contribution at high energy comes from

$$
T_{\Psi}^{\mu \nu} \propto 2\left(p^{\mu} p^{\prime \nu}+p^{\nu} p^{\prime \mu}\right) e^{-i\left(p-p^{\prime}\right) \cdot x} z^{5}\left(J_{\tau-2}^{2}\left(M z^{\prime}\right)+J_{\tau-1}^{2}\left(M z^{\prime}\right)\right)+\cdots .
$$

Thus the $t$-channel graviton exchange amplitude for the dilatino is

$$
\begin{aligned}
\mathcal{T}_{\mu \nu}^{G \Psi} \propto & \mathcal{A}^{\prime \prime}\left[\left(p_{\mu}+\frac{q_{\mu}}{2 x}\right)\left(p_{\nu}^{\prime}+\frac{q_{\nu}^{\prime}}{2 x^{\prime}}\right)+\left(p_{\mu}^{\prime}-\frac{q_{\mu}}{2 \tilde{x}}\right)\left(p_{\nu}-\frac{q_{\nu}^{\prime}}{2 \tilde{x}^{\prime}}\right)\right] q^{2} q^{\prime 2} \mathcal{T}_{1}^{G \Psi} \\
& +\mathcal{A}^{\prime \prime}\left[\begin{array}{c}
\frac{q^{\prime} q}{4 x x^{\prime}}\left(\eta_{\mu \nu}+\frac{2 x}{q^{2}} p_{\mu} q_{\nu}+\frac{2 x^{\prime}}{q^{\prime 2}} q_{\mu}^{\prime} p_{\nu}^{\prime}+\frac{4 x x^{\prime}}{q^{2} q^{\prime 2}} q \cdot q^{\prime} p_{\mu} p_{\nu}^{\prime}\right) \\
+\frac{q^{\prime} q}{4 \tilde{x} \tilde{x}^{\prime}}\left(\eta_{\mu \nu}-\frac{2 \tilde{x}}{q^{2}} p_{\mu}^{\prime} q_{\nu}-\frac{2 \tilde{x}^{\prime}}{q^{\prime 2}} q_{\mu}^{\prime} p_{\nu}+\frac{4 \tilde{x} \tilde{x}^{\prime}}{q^{\prime} q^{\prime}} q \cdot q^{\prime} p_{\mu}^{\prime} p_{\nu}\right)
\end{array}\right] q^{2} q^{\prime 2} \mathcal{T}_{2}^{G \Psi},
\end{aligned}
$$

with

$$
\begin{aligned}
\mathcal{T}_{1}^{G \Psi}= & \int d z z^{3} K_{0}(q z) K_{0}\left(q^{\prime} z\right) \int d z^{\prime} z^{\prime 3}\left(J_{\tau-2}^{2}\left(M z^{\prime}\right)+J_{\tau-1}^{2}\left(M z^{\prime}\right)\right) \\
& \times \int_{\mathcal{C}} \frac{d j}{2 i} \frac{1+e^{-i \pi j}}{\sin \pi j} \frac{\left(\alpha^{\prime} s\right)^{j-2+\alpha^{\prime} t z^{\prime 2}}}{\sqrt{j-j_{0}}}\left(\frac{z^{2}}{z^{\prime 2}}\right)^{1-\gamma}, \\
\mathcal{T}_{2}^{G \Psi}= & \int d z z^{3} K_{1}(q z) K_{1}\left(q^{\prime} z\right) \int d z^{\prime} z^{\prime 3}\left(J_{\tau-2}^{2}\left(M z^{\prime}\right)+J_{\tau-1}^{2}\left(M z^{\prime}\right)\right) \\
& \times \int_{\mathcal{C}} \frac{d j}{2 i} \frac{1+e^{-i \pi j}}{\sin \pi j} \frac{\left(\alpha^{\prime} s\right)^{j-2+\alpha^{\prime} t z^{\prime 2}}}{\sqrt{j-j_{0}}}\left(\frac{z^{2}}{z^{\prime 2}}\right)^{1-\gamma} .
\end{aligned}
$$

The evaluation of the above Compton scattering amplitude is quite complicated. Nevertheless, we can approximately find the corresponding DVCS cross section for both dilaton and dilatino targets at the $t=0$ limit is

$$
\frac{d \sigma_{\mathrm{G}}^{\mathrm{Un}}}{d t} \propto \frac{1}{s^{2}}\left(s^{2-\frac{2}{\sqrt{\lambda}}}\right)^{2}\left(\frac{M^{2}}{Q^{2}}\right)^{2},
$$

where the factor $\frac{1}{s^{2}}$ comes from the phase space integral and $s^{2-\frac{2}{\sqrt{\lambda}}}$ arises due to the graviton exchange together with its curvature correction. The energy enhancement in Eq. (167) indicates that the $t$-channel graviton exchange should dominate the total scattering amplitude in the high-energy limit. 


\section{CONCLUSION}

In this paper, we calculate the supergravity graphs corresponding to Compton scatterings for both dilaton and dilatino targets in the CFT. For a dilaton target, we have calculated the $s$-channel, $u$-channel graphs where all the Kaluza-Klein excitations of intermediate states are summed over, and the four-point contact term. We sum all these three graphs and compute the usual real Compton scattering amplitude and DVCS cross section. For a dilatino target, we compute the contributions from the $s$-channel and $u$-channel graphs explicitly. We find that the real Compton scattering amplitude, up to an overall constant, is identical to the amplitude found in scalar QED. The DVCS cross section is also discussed afterwards and compared to the low-energy $\mathrm{H} 1$ and ZEUS data. The curve is consistent with the experimental data. The end of this paper is devoted to discussing the $t$-channel graviton exchange contribution, which is dominant contribution in the high-energy limit. We have provided a heuristic derivation for the computation of the $t$-channel graviton exchange.

It will be interesting to continue this study and extract the generalized parton distributions as well as the information about the total spin of constituents in a hadron. This would help us to understand the role of the orbital angular momentum and the spin sum rule at strongly coupled regime.

\section{Acknowledgments}

We acknowledge inspiring discussions with E. Iancu, Y. Hatta, C. Marquet, A. H. Mueller and F. Yuan. J. G. acknowledges financial support by the China Postdoctoral Science Foundation funded project under Contract No. 20090460736. B. X. is supported by the Director,

Office of Energy Research, Office of High Energy and Nuclear Physics, Divisions of Nuclear Physics, of the U.S. Department of Energy under Contract No. DE-AC02-05CH11231.

[1] J. M. Maldacena, Adv. Theor. Math. Phys. 2, 231 (1998) [Int. J. Theor. Phys. 38, 1113 (1999)] arXiv:hep-th/9711200.

[2] E. Witten, Adv. Theor. Math. Phys. 2, 253 (1998) arXiv:hep-th/9802150. 
[3] S. S. Gubser, I. R. Klebanov and A. M. Polyakov, Phys. Lett. B 428, 105 (1998) arXiv:hep-th/9802109].

[4] J. Polchinski and M. J. Strassler, Phys. Rev. Lett. 88, 031601 (2002) arXiv:hep-th/0109174.

[5] J. Polchinski and M. J. Strassler, JHEP 0305, 012 (2003) [arXiv:hep-th/0209211].

[6] R. C. Brower and C. I. Tan, Nucl. Phys. B 662, 393 (2003) arXiv:hep-th/0207144.

[7] H. Boschi-Filho and N. R. F. Braga, Phys. Lett. B 560, 232 (2003) arXiv:hep-th/0207071.

[8] R. C. Brower, M. J. Strassler and C. I. Tan, JHEP 0903, 092 (2009) [arXiv:0710.4378 [hep-th]].

[9] R. C. Brower, J. Polchinski, M. J. Strassler and C. I. Tan, JHEP 0712, 005 (2007) arXiv:hep-th/0603115].

[10] C. A. Ballon Bayona, H. Boschi-Filho and N. R. F. Braga, JHEP 0809, 114 (2008) arXiv:0807.1917 [hep-th]].

[11] C. A. Ballon Bayona, H. Boschi-Filho and N. R. F. Braga, JHEP 0810, 088 (2008) arXiv:0712.3530 [hep-th]].

[12] C. A. Ballon Bayona, H. Boschi-Filho and N. R. F. Braga, JHEP 0803, 064 (2008) arXiv:0711.0221 [hep-th]].

[13] B. Pire, C. Roiesnel, L. Szymanowski and S. Wallon, Phys. Lett. B 670, 84 (2008) arXiv:0805.4346 [hep-ph]].

[14] L. Cornalba and M. S. Costa, Phys. Rev. D 78, 096010 (2008) arXiv:0804.1562 [hep-ph]].

[15] J. L. Albacete, Y. V. Kovchegov and A. Taliotis, JHEP 0807, 074 (2008) arXiv:0806.1484 [hep-th]].

[16] E. Levin, J. Miller, B. Z. Kopeliovich and I. Schmidt, JHEP 0902, 048 (2009) arXiv:0811.3586 [hep-ph]].

[17] Y. V. Kovchegov, Z. Lu and A. H. Rezaeian, Phys. Rev. D 80, 074023 (2009) arXiv:0906.4197 [hep-ph]].

[18] Y. Hatta, E. Iancu and A. H. Mueller, JHEP 0801, 063 (2008) arXiv:0710.5297 [hep-th]].

[19] A. H. Mueller, A. I. Shoshi and B. W. Xiao, Nucl. Phys. A 822, 20 (2009) arXiv:0812.2897 [hep-th]].

[20] E. Avsar, E. Iancu, L. McLerran and D. N. Triantafyllopoulos, JHEP 0911, 105 (2009) arXiv:0907.4604 [hep-th]].

[21] F. Dominguez, arXiv:0912.1641 [hep-th].

[22] C. A. B. Bayona, H. Boschi-Filho and N. R. F. Braga, arXiv:0912.0231 [hep-th]. 
[23] E. Iancu and A. H. Mueller, arXiv:0912.2238 [hep-th].

[24] Y. Hatta, E. Iancu and A. H. Mueller, JHEP 0801, 026 (2008) [arXiv:0710.2148 [hep-th]].

[25] F. Dominguez, C. Marquet, A. H. Mueller, B. Wu and B. W. Xiao, Nucl. Phys. A 811, 197 (2008) arXiv:0803.3234 [nucl-th]].

[26] J. H. Gao and B. W. Xiao, Phys. Rev. D 80, 015025 (2009) arXiv:0904.2870 [hep-ph]].

[27] Y. Hatta, T. Ueda and B. W. Xiao, JHEP 0908, 007 (2009) arXiv:0905.2493 [hep-ph]].

[28] X. D. Ji, Phys. Rev. Lett. 78, 610 (1997) arXiv:hep-ph/9603249.

[29] D. Mueller, D. Robaschik, B. Geyer, F. M. Dittes and J. Horejsi, Fortsch. Phys. 42, 101 (1994) arXiv:hep-ph/9812448.

[30] X. D. Ji, Phys. Rev. D 55, 7114 (1997) arXiv:hep-ph/9609381.

[31] A. V. Radyushkin, Phys. Lett. B 380, 417 (1996) arXiv:hep-ph/9604317.

[32] A. V. Radyushkin, Phys. Rev. D 56, 5524 (1997) arXiv:hep-ph/9704207].

[33] X. Ji, Ann. Rev. Nucl. Part. Sci. 54, 413 (2004).

[34] A. V. Belitsky and A. V. Radyushkin, Phys. Rept. 418, 1 (2005) arXiv:hep-ph/0504030.

[35] K. Goeke, M. V. Polyakov and M. Vanderhaeghen, Prog. Part. Nucl. Phys. 47, 401 (2001) arXiv:hep-ph/0106012.

[36] M. Diehl, Phys. Rept. 388, 41 (2003) arXiv:hep-ph/0307382.

[37] S. J. Brodsky, M. Diehl and D. S. Hwang, Nucl. Phys. B 596, 99 (2001) arXiv:hep-ph/0009254.

[38] H. Liu and A. A. Tseytlin, Phys. Rev. D 59, 086002 (1999) arXiv:hep-th/9807097.

[39] D. Z. Freedman, S. D. Mathur, A. Matusis and L. Rastelli, Phys. Lett. B 452, 61 (1999) arXiv:hep-th/9808006.

[40] E. D'Hoker, D. Z. Freedman, S. D. Mathur, A. Matusis and L. Rastelli, Nucl. Phys. B 562, 353 (1999) arXiv:hep-th/9903196.

[41] J. Bartels, J. Kotanski, A. M. Mischler and V. Schomerus, arXiv:0908.2301 [hep-th].

[42] G. F. de Teramond and S. J. Brodsky, Phys. Rev. Lett. 94, 201601 (2005) arXiv:hep-th/0501022.

[43] S. J. Brodsky and G. F. de Teramond, Phys. Rev. Lett. 96, 201601 (2006) arXiv:hep-ph/0602252.

[44] M. Henningson and K. Sfetsos, Phys. Lett. B 431, 63 (1998) arXiv:hep-th/9803251.

[45] W. Mueck and K. S. Viswanathan, Phys. Rev. D 58, 041901 (1998) arXiv:hep-th/9804035. 
[46] A. Aktas et al. [H1 Collaboration], Eur. Phys. J. C 44, 1 (2005) arXiv:hep-ex/0505061].

[47] S. Chekanov et al. [ZEUS Collaboration], Phys. Lett. B 573, 46 (2003) arXiv:hep-ex/0305028].

[48] F. D. Aaron et al. [H1 Collaboration], Phys. Lett. B 659, 796 (2008) arXiv:0709.4114 [hep$\mathrm{ex}]]$.

[49] J. Polchinski, String Theory: An Introduction to the Bosonic String (Cambridge University Press, Cambridge, England, 1998),Vol. 1, p. 402. 